\title{
[L్
}

LAWFENCE INERMORE LABORATOAY

Universityor Calionia/Livermore, California/94550

UCRL-51233

\section{AN EXAMINATION OF RAYLEIGH WAVES PRODUCED BY SHEAR AND COMPRESSIONAL LINE SOURCES}

\author{
W. J. Hannon
}

June 2, 1972

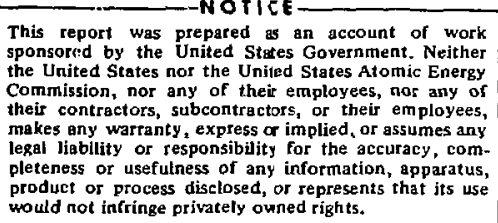

would not infringe privately ouned rights. 


\section{Contents}

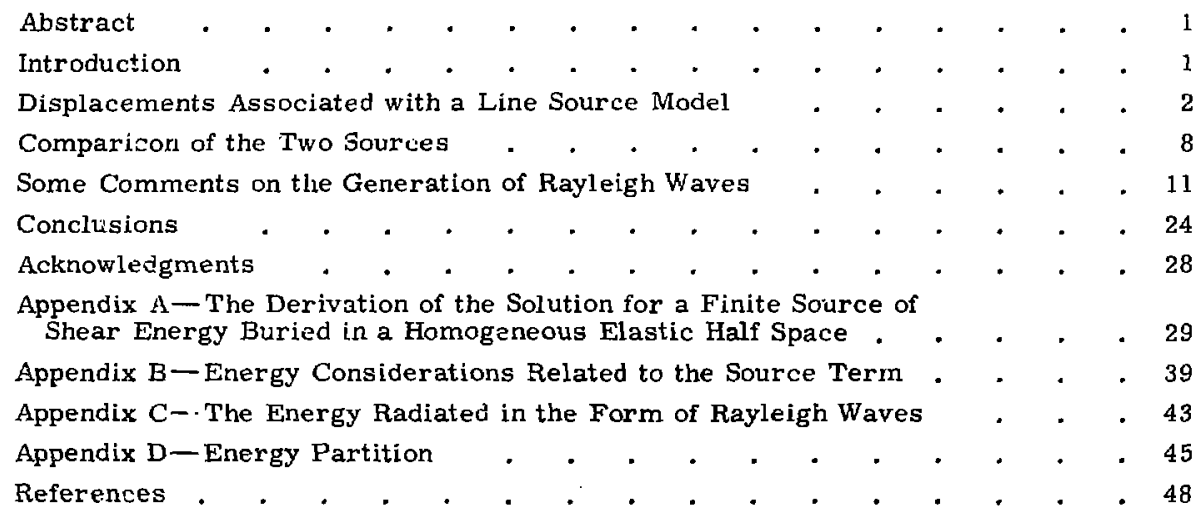




\title{
AN EXAMINATION OF RAYLEIGH WAVES PRONUCED BY SHEAR AND COMPRESSIONAL LINE SOURCES
}

\begin{abstract}
This article considers the response of a semi-infinite homogeneous medium to shear and compressional line sources. Various energy and energy density functions are obtained from the expressions for the displacements as a function of time and position. Comparison of the results for the two sources indicates that the shear source is a better generator of Rayleigh waves than is the compressional source. Depending on the normalization used, a factcr of between 3 and 30 is appropriate for Poisson's ratios typical of rocks. This suggests that the surface wave magnitude versus body wave magnitude discrimination technique used in seismic identification may be affected by the use of shaped cavities or other approaches which enhance the generation of shear waves by explosions.

The distribution of enerr,y density as a function of time and position indicates that in a homogeneous half space the immediate sources of energy for the Rayleigh wave are the energy radiated from late time distortional energy concentrations above the source and the energy from regions where the reflected shear waves are large. The energy from these source regions interacts with the free surface to form the Rayleigh wave.
\end{abstract}

\section{Introduction}

At the present time, one of the important criteria used to distinguish underground nuclear explosions from earthquakes is the comparison of the seismic magnitude of the event deternined from surface waves with the seismic magnitude determined by body waves. In general, if an earthquake and an explosion have the same body wave magnitude $\left(m_{b}\right)$ the explcsion will have a smaller surface wave magnitude $\left(M_{s}\right)$ than the earthquake. This fact is the basis for the use of the two magnitudes in discrimination techniques.

Since the explosion is, ordinarily, primarily a source of compressional waves, whereas the earthquake is a source of both shear and compressional waves, the question arises as to whether techniques which would cause an explosion to initiate shear energy as well as compressional energy in the source region would significantly reduce he usefulness of the $\mathrm{M}_{\mathrm{s}}-\mathrm{m}_{\mathrm{b}}$ discriminant criterion. This possibility was suggested by Rodean. ${ }^{1}$ Further indications that shear and compressional sources have different efficiencies in generating surface waves (Rayleigh waves, in this case) were found in 
an article by Nakano $\overline{ }$ in which he considered Rayleigh waves arising from simple harmonic shear and compressional line isources buried in an elastic, homogene Jus half spa.ce.

Since various mechanisms (e.g., multiple sources, non-spheriçal cavities, inhomogeneous media) are availabie to affect the amount of shear enerE $y$ erritted from the source region, we decided to determine the relationship between the energy generated by shear and compressional sources and the energy contained in the associated Rayleigh waves. In order to achieve some degree of comoutational simplicity we considered aperiodic compressional and shear line sources buried in a homogeneous elastic half-space.

\section{Displacements Associated with a Line Source Model}

Many authors have considered various aspects of the response of the medium to these sources (see for example the bibliography for Chapter 2 in Ewing et al. ${ }^{3}$ for some of the early references). Most of these investigations dealt with periodic time variations or obtained results which were primarily applicable in the far field. Garvin ${ }^{4}$ obtained closed form expressions for the free surface displacements from a buried compressional line source at all times. The source was formulated in terms of a particular displacement potential whose form leut itself to the application of Cagniard's technique ${ }^{5}$ for evaluating the inverse Laplace transforms appearing in the analysis of the problem. The corresponding body wave displacements begin with an infinite amplitude. Since Garvin's initial work, other investigators ${ }^{6-10}$ have used related techniques to study various aspects of the displacement field. In these articles it is possible to find rorms which describe:

a. the displacements at all points in and on the surface of the half-space which ari ${ }^{i}$ from a buried compressional source

b. the displacements at the free surface which arise from a buried shear source.

In this paper we follow Garvin's approach ${ }^{4}$ and give results which will describe the displacements at all points in the medium arising from either source. From the displacements we can determine the strains and the energy flux and thus compare the two sources in a variety of ways. Figure 1 illustrates the geometry for the model. The line source is oriented parallel to the $\mathrm{z}$ axis and is located at a distance $\mathrm{h}$ from the free sur face of a homogeneous elastic half-space.

Initially the medium is at rest. At some time, the line source disturbs the medium by emitting an axially symmetric pulse. The source time history for the displacement can be described as a sudden enset followed by a gradual, incomplete recovery (Fig. 2).

Although the solution for the shear-wave line source is in many ways similar to that for the compressional line source, the full solutions applicable to any point in

$$
-2-
$$



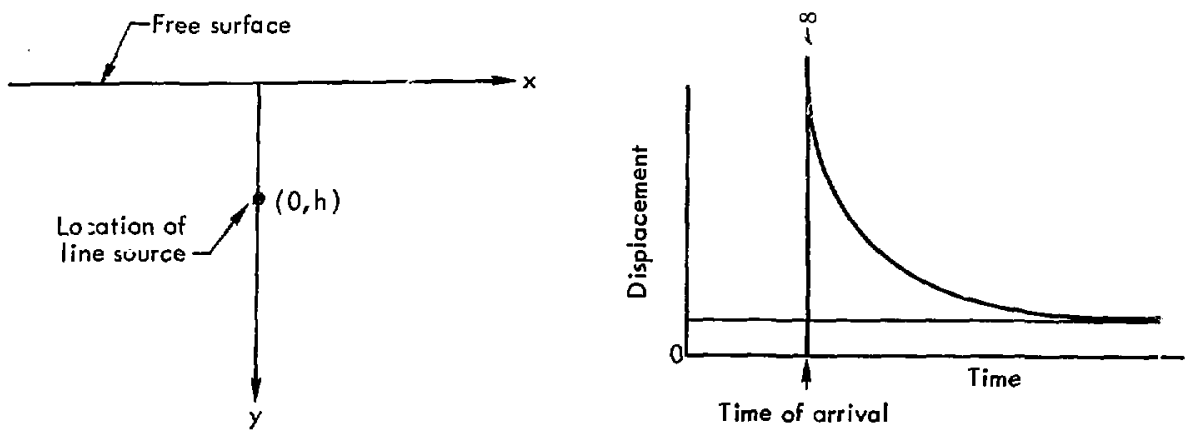

Figure 1. The geometry of the problem. Figure 2. The displacement time history..

space do not appear to have been jublished. We discuss the derivation of these sclutions in Appendix A. In Eqs. (1) and (2) we give the expressions for the vertical (s $y$ ) and horizontal $\left(s_{x}\right)$ components of displacement at all points of the semi-finite medium for both compressional and shear-wave line sources. The components of the displacement for the two sources are given in terms of distances scaled to the depth of burial and times scaled to the time required for the source wave to travel to the free surface.

For a compressional source, the displacements are:

$$
\begin{aligned}
& \mathrm{hs}_{\mathrm{cx}}=\frac{\operatorname{an} \xi}{2 \theta_{-}^{2}} \frac{1}{\sqrt{\eta^{2}-\theta_{-}^{2}}} \mathrm{H}\left(\eta-\sigma_{-}\right)-\frac{\operatorname{an} \xi}{2 \theta_{+}^{2}} \frac{1}{\sqrt{\eta^{2}-\theta_{+}^{2}}} \mathrm{H}\left(\eta-\theta_{+}\right) \\
& +\operatorname{Im}\left\{\frac{4 a b^{3} v_{3}^{3} \sqrt{1+b^{2} v_{3}^{2}}}{\theta_{+}^{2} \Delta_{c}\left(b, U_{3}^{2}\right)}\left[-i \xi+\frac{(1+\xi) \eta}{\left(\eta^{2}-\theta_{+}^{2}\right)^{1 / 2}}\right]\right\}\left(\eta-\theta_{+}\right) \\
& -\operatorname{Im}\left\{\frac{2 a b U_{4} \sqrt{1+b^{2} \mathrm{U}_{4}^{2}}\left(1+2 b^{2} \mathrm{U}_{4}^{2}\right)}{\Delta_{\mathrm{c}}\left(\mathrm{b}, \mathrm{U}_{4}^{2}\right)\left[\mathrm{i} \xi+\frac{\mathrm{U}_{4}}{\sqrt{1+\mathrm{U}_{4}^{2}}}+\frac{\zeta \mathrm{U}_{4}}{\sqrt{\frac{1}{\mathrm{~b}} 2+\mathrm{U}_{4}^{2}}}\right]}\right\} \mathrm{H}\left(n-n_{\mathrm{s}}\right)
\end{aligned}
$$

$h \Sigma_{c y}=-\frac{a(1-\zeta) \eta}{2 \theta_{-}^{2}} \frac{1}{\sqrt{\eta^{2}-\theta_{-}^{2}}} \quad \mathrm{H}\left(\eta-\theta_{-}\right)-\frac{\mathrm{a}(1+\zeta) \eta}{2 \theta_{+}^{2}} \frac{1}{\sqrt{\eta^{2}-\theta_{+}^{2}}} \mathrm{H}\left(\eta-\theta_{+}\right)$ 


$$
\begin{aligned}
& -\operatorname{Re}\left\{\frac{4 a b^{3} \mathrm{U}_{3}^{2} \sqrt{1+\mathrm{u}_{3}^{2}} \sqrt{\mathrm{i}+\mathrm{b}^{2} \mathrm{U}_{3}^{2}}}{\theta_{+}^{2} \Delta_{\mathrm{c}}\left(\mathrm{b}, \mathrm{U}_{3}^{2}\right)}\left[-\mathrm{i} \xi+\frac{(1+\zeta) \eta}{\left(\eta^{2}-\theta_{+}^{2}\right)^{1 / 2}}\right]\right\} H\left(\eta-\theta_{+}\right) \\
& +\operatorname{Re}\left\{\frac{2 a \mathrm{U}_{4}^{2} \mathrm{~b}^{2}\left(1+2 \mathrm{~b}^{2} \mathrm{r}_{4}^{2}\right)}{\Delta_{\mathrm{c}}\left(\mathrm{b}, \mathrm{U}_{4}^{2}\right)\left(\mathrm{i} \xi+\frac{\mathrm{U}_{4}^{4}}{\sqrt{1+\mathrm{U}_{4}^{2}}}+\frac{\mathrm{b} \zeta \mathrm{U}_{4}}{\sqrt{1+\mathrm{b}^{2} \mathrm{U}_{4}^{2}}}\right)}\right\} \mathrm{H}\left(\eta-\eta_{\mathrm{s}}\right)
\end{aligned}
$$

where

$s_{c x} \cdot s_{c y}$ are the horizontal $(x)$ and vertical $(y)$ components of the displacement due to the compressional source,

$$
\begin{aligned}
& a=\text { the strength of the source, } \\
& t=\text { time, } \\
& x_{,} y=\text { coordinates of the field point, } \\
& h=\text { the depth of burial of the source } \\
& r_{ \pm}^{2}=x^{2}+(y \pm h)^{2}, \\
& V_{s}=\text { velocity of shear waves, } \\
& V_{c}=\text { velocity of compressional waves, } \\
& b=V_{s} / V_{c} \\
& \eta \quad=\text { dimensionless time }=V_{c} t / h, \\
& \xi \quad=\text { dimensionless } x \text { coordinate }=x / h, \\
& \zeta \quad=\text { dimensionless } y \text { coordinate }=y / h, \\
& { }_{ \pm}=r_{ \pm} / h,
\end{aligned}
$$$$
\mathrm{H}(\mathrm{z})=0 \mathrm{z}<0
$$$$
=1 z>0 \text {, }
$$$$
U_{3}=\frac{-i \eta \xi+(1+\zeta)\left(\eta^{2}-\theta_{+}^{2}\right)^{1 / 2}}{\theta_{+}^{2}} \text {. }
$$

$\mathrm{U}_{\mathbf{i}}=$ is defined implicitly by

$$
\eta=\mathrm{iU}_{4} \xi+\sqrt{1+\mathrm{U}_{4}^{2}}+\frac{\zeta}{\mathrm{b}} \sqrt{1+\mathrm{b}^{2} \mathrm{U}_{4}^{2}},
$$

$\eta_{\mathrm{s}}$ is the above $\eta$ evaluated at $\mathrm{U}_{4}=\mathrm{iV}$ where $\mathrm{V}$ satisfies the equation

$$
\xi-\frac{\mathrm{V}}{\sqrt{1-\mathrm{v}^{2}}}-\frac{\zeta \mathrm{Vb}}{\sqrt{1-\mathrm{b}^{2} \mathrm{v}^{2}}}=0 \text {. }
$$

and

$$
\Delta_{c}\left(b, u^{2}\right)=\left(1+2 b^{2} u^{2}\right)^{2}-4 b^{3} u^{2} \sqrt{1+u^{2}} \sqrt{1+b^{2} u^{2}} .
$$


The corresponding equations for the shear source are:

$$
\begin{aligned}
& h s_{\mathrm{sx}}=\frac{\mathrm{a}(1-\xi)}{2 \theta_{-}^{2}} \frac{\eta^{\prime}}{\sqrt{\eta^{\prime}{ }^{2}-\theta_{-}^{2}}} \mathrm{H}\left(\eta^{\prime}-\theta_{-}\right)+\frac{\mathrm{a}(1+\xi)}{2 \theta_{+}^{2}} \frac{\eta^{\prime}}{\sqrt{\eta^{\prime}{ }^{2}-\theta_{+}^{2}}} \mathrm{H}\left(\eta^{\prime}-\theta_{+}\right) \\
& +\operatorname{Re}\left\{\frac{4 \mathrm{aU}_{3}^{2} \sqrt{\mathrm{b}^{2}+\mathrm{UI}_{3}^{2}} \sqrt{1+\mathrm{U}_{3}^{12}}}{\theta_{+}^{2} \Delta_{\mathrm{s}}\left(\mathrm{b}, \mathrm{U}_{3}^{\prime 2}\right)}\left[-\mathrm{i} \xi+\frac{(1+\zeta) \eta^{\prime}}{\left(\eta^{12}-\theta_{+}^{2}\right)^{1 / 2}}\right] \mathbf{H}^{\prime}\left(\eta^{\prime}-\eta_{\text {choice }}\right)\right. \\
& -\operatorname{Re}\left\{\frac{2 a U_{4}^{2}\left(1+2 U_{4}^{2}\right)}{\Delta_{S}\left(b, U_{4}^{\prime 2}\right)} \frac{1}{\left[i \xi+\frac{U_{4}^{\prime}}{\sqrt{1+U_{4}^{2}}}+\frac{\zeta U_{4}^{\prime}}{\sqrt{b^{2}+U_{4}^{2}}}\right]}\right\} H\left(\eta^{\prime}-\eta_{p}\right)
\end{aligned}
$$

$$
\begin{aligned}
& h_{\text {is }}=\frac{a \xi}{2 \theta_{-}^{2}} \frac{\eta^{\prime}}{\sqrt{\eta^{\prime}{ }^{2}-\theta_{-}^{2}}} \quad H\left(\eta^{\prime}-\theta_{-}\right)-\frac{a \xi}{2 \theta_{+}^{2}} \frac{\eta^{\prime}}{\sqrt{\eta^{\prime 2}-\theta_{+}^{2}}} \quad H\left(\eta^{\prime}-\theta_{+}\right) \\
& +\operatorname{Im}\left\{\frac{4 a U_{3}^{3} \sqrt{b^{2}+I_{3}^{2}}}{\theta_{+}^{2} \Delta_{s}\left(b, U_{3}^{2}\right)}\left[-i \xi+\frac{(1+\zeta) \eta}{\left(\eta^{2}-\theta_{+}^{2}\right)^{1 / 2}}\right]\right\} \quad \mathrm{F}\left(\eta^{\prime}-\eta_{c h o i c e}\right)
\end{aligned}
$$

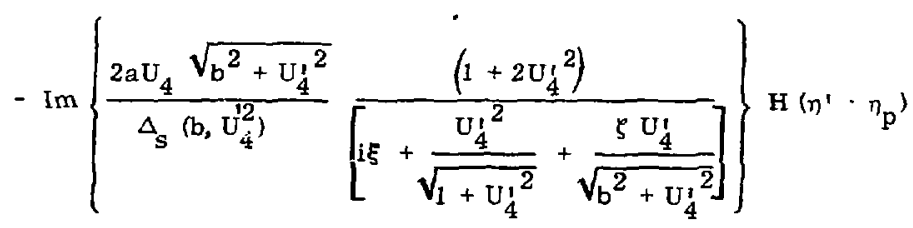

where

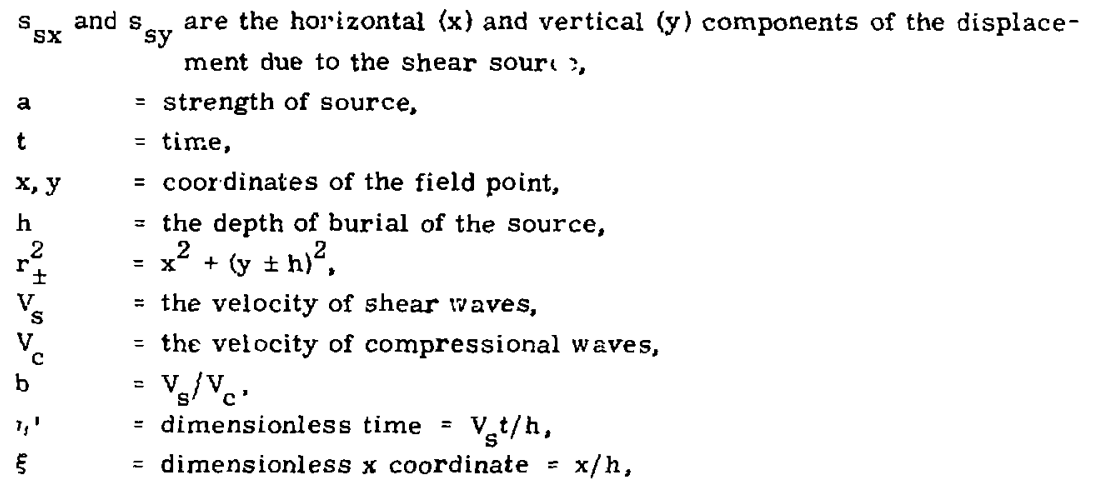




$$
\begin{aligned}
& \zeta=\text { dimensionless } \mathrm{y} \text { coordinate }=\mathrm{y} / \mathrm{h} \text {, } \\
& \theta_{t} \quad=r_{t} / \mathrm{h} \\
& \mathrm{H}(\mathrm{z}) \quad=0 \mathrm{z}<0 \\
& 1 z>0 \\
& \mathrm{U}_{3}^{\prime}=\frac{-i \eta \xi+(1+\zeta) \sqrt{\eta^{\prime 2}-\theta_{+}^{2}}}{\theta_{+}^{2}} \text {. } \\
& n_{\text {choice }}=\theta_{+} \text {if } \xi / \theta_{+} \leq \mathrm{b} \\
& =b \xi+(1+\zeta) \sqrt{1-b^{2}} \text { if } \xi / \theta_{+}>b,
\end{aligned}
$$

$\mathrm{U}_{4}^{\prime}$ is defined implicitly by

$$
n^{\prime}=\mathrm{i} \xi \mathrm{U}_{4}^{\prime}+\zeta \sqrt{\mathrm{b}^{2}+\mathrm{U}_{4}^{2}}+\sqrt{1+\mathrm{U}_{4}^{2}},
$$

$\eta_{\mathrm{p}}$ is the above evaluated at $\mathrm{U}_{4}^{\prime}=-\mathrm{i} \mathrm{V}^{\prime}$ where $\mathrm{V} \cdot$ satisfies the equations

and

$$
\begin{aligned}
\xi-\frac{\zeta V^{\prime}}{\sqrt{b^{2}-V^{2}}}-\frac{V^{\prime}}{\sqrt{1-V^{\prime 2}}}=0, & \zeta \neq 0 \\
V^{\prime}=b & \zeta=0
\end{aligned}
$$

$$
\Delta_{s}\left(b, U^{2}\right)=\left(1+2 U^{2}\right)^{2}-4 U^{2} \sqrt{1+U^{2}} \sqrt{b^{2}+U^{2}}
$$

With the exceptio: 1 of the fact that $\mathrm{U}_{4}, \mathrm{U}_{4}^{1}, n_{\mathrm{s}}$, and $n_{\mathrm{p}}$ are defined implicitly, "hese equations represent closed-form solutions to the shear and compressional line-source problems. The implicit definitions of the above quantities post no computational problems, and so the displacements may be evaluated numerically as functions of $\xi$, and $\eta$ by means of the above expressions and the numerical solutions of the quartic and cubic equations associated with $U_{4}, U_{4}^{\prime}$, and $\eta_{p}, \eta_{s}$ respectively.

Some examples of the displacements arising from the shear and compressional sources are shown in Fig. 3. Li Figs. $3 a$ and $3 b$ we see the displacements at the surface near the source. The waveforms are simple and similar to the waveform of the source function (Fig. 2). Sirice the displacements corresponding to this source begin with an infinite discontinuity, it is not possible to assign any significance to the absolute amplitudes of the components in these and the following figures. However, two points are worth noting for Figs. $3 \mathrm{a}$ and $3 \mathrm{~b}$ :

1. The relative amplitudes of the components for a single event give a measure of the apparent angle of incidence (see Ewing et al. ${ }^{3}$ ).

2. The displacements at this distance show a displacement away frorn the rest position at late times.

The latter fact plays an important part in discussions of mechanisms of Rayleigh wave generation (see Mateker, ${ }^{11}$ for example). In Figs. $3 \mathrm{c}$ and $3 \mathrm{~d}$, we see the development 

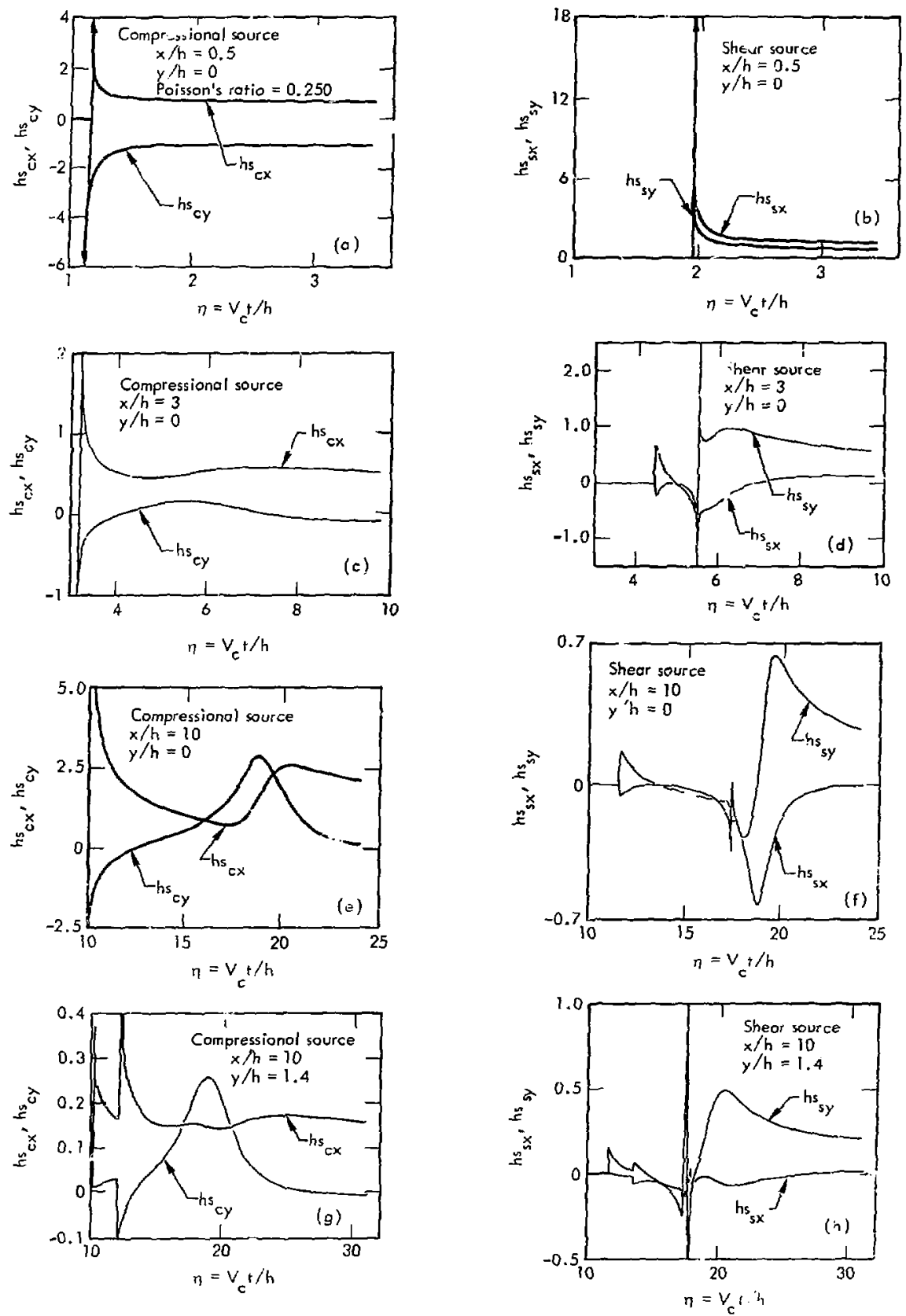

Figure 3. Representative displacements for the shear and compressional sources as calculatec fron Eqs. (1) and (2). Figures a-f are similar to those of Takeuchi and kobayashi.? 
of the $\mathrm{Ra}_{\mathrm{v}}$ : igh wave at a distance $\xi=3.0$ at about $\eta=5.5$ for the compressional source, and slightly later for the shear source. Also, in Fig. 3d we see the arrival of a precursor at $\eta=4.5$ in acidition to the shear wave and Rayleigh wave. This wave is associated with the critical angle reflection phenomena that occur when the shear wave strikes the free surface. Figures $3 e$ and $3 f$ are similar to these, but for a distarce $\xi=10.0$. At this distance the Rayleigh waves are well defined. We note that the roles of the horizontal and vertical components are interchanged for the two sources. This is related to the fact that in the surface region near the source where the Rayleigh waves are generated, the motion from the shear source is predominantly horizontal while that from the compressional source is predominantly vertical. We alsc note that amplitude of the Rayleigh wave from the shear source is about three times as large as that from the compressional source. Figures $3 \mathrm{~g}$ and $3 \mathrm{~h}$ show the displacements at the sam $z$ horizontal distance as.Figs. $3 e$ and $3 f$. iowever, in this case the field point is at a depth of 1.4 times the depth of burial. Both the compressionai and shear sources show that the Rayleigh wave has become prograde. The results for the shear source are quite complex and show four arrivals in addition to the Rayleigh wave. These are, in the order of increasing time: the reflected $\mathbf{P}$ wave, the curical $S$ wave, 5 the direct $S$ wave, and the reflected $S$ wave.

In each of the expressions for the displacements given in Eqs. (1) and (2), the first term contributes to the direct wave and the second term to the reflected wave. The third term constitutes a correction to the reflected wave and includes the conical wave (where appropriate) for the shear source. The fourth term represents the converted reflected wave (e.g., compressional sources generate reflected shear waves).

\section{Comparison of the Two Sources}

Alti. " "Th the models chosen have the advantage that the displacements are easily calculated, the comparison of the relative efficiencies of the two sources as generators of Payleigh waves is made difficult by the fact that the displacements associated with the direct and the reflected waves begin with an infinite discontimuity. Also, we are faced with the problem that the tirne scales in the two problems are different, i.e., the compressional wave solution is expressed in terms of $\eta=\left(V_{s} t / h\right)$ whereas the shear wave solution is expressed in terms of $\eta^{\prime}=\left(V_{s} t / h\right)$. However, we can use the similarities of the source expressions to make a comparison between the two sources.

As we pointed out above, the first terms in the equations represent the contribution of the source. If we examine the terms, we find

$$
\left|\mathrm{hs}_{\mathrm{c}_{1}}\right|=\sqrt{\left(\mathrm{hs_{ \textrm {cx } }}\right)^{2}+\left(\mathrm{hs}_{\mathrm{cy}}\right)^{2}}=\frac{\mathrm{a}}{2 \theta}-\frac{\eta}{\sqrt{\eta^{2}-\theta_{-}^{2}}} \text {. }
$$


Similarly,

$$
\left|h s_{s_{1}}\right|=\sqrt{\left(h_{s x_{1}}\right)^{2}+\left(h_{s y_{1}}\right)^{2}}=\frac{a}{2 \theta_{-}} \frac{\eta^{\prime}}{\sqrt{n^{\prime 2}-\theta_{-}^{2}}} .
$$

Thus, if we compare the displacement fields from the two sources at the same values of the scaled times, we see that the magnitude of the displacements at a given field point are the same. Thus, in this sense, we can consider the amplitudes of the two sources to be the same.

Another possible way to compare the two sources is to consider the energy radiated by them if they were located in an infinite medium. In Appendix $B$, we see that as the two scaled times and thus the two amplitudes change by the same amount, the ratio of the energy radiated into the far field by the compressional source to the energy radiated by the shear source is $\mathrm{v}_{\mathrm{p}}^{2} / \mathrm{v}_{\mathrm{s}}^{2}$. In this serse, the dilatational source is the stronger source. Finally, in Appendix B, we also consider the residual strain energy. We see that the residual strain energy in the medium is the same for both sources. This is not surprising when we realize that are residual energy is primarily distortional energy in both cases.

Thus we see that if we make allowance for the differences in decay rate, the waveforms of the two sources are identical as are the residual strain energies. The compressional source is a stronger dynamic scurce in the sense that it radiates more energy into the far field.

Having established the relative properties of the sources, we can ccnsider the Rayleigh waves generated by these sources in a semi-infinite half space. Table 1 lists some represencative amplitudes obtained from calculations similar to those giving rise to Fig. 3 .

Table 1. Peak-to-peak amplitudes of Rayleigh waves from shear and compressional line sources of the Garvin 4 type.

\begin{tabular}{lllll}
\hline \multirow{2}{*}{$\begin{array}{c}\text { Poisson's } \\
\text { ratio }\end{array}$} & \multicolumn{2}{c}{ Compressional source } & \multicolumn{2}{c}{ Shear source } \\
\cline { 2 - 5 } & hs $_{\mathrm{cx}}$ & hs $_{\mathrm{cy}}$ & $\mathrm{hs}_{\mathrm{sx}}$ & $\mathrm{hs}_{\mathrm{sy}}$ \\
\hline 0.10 & 0.34 & 0.45 & 0.80 & 1.0 \\
0.15 & 0.29 & 0.39 & 0.74 & 1.0 \\
0.20 & 0.24 & 0.34 & 0.68 & 0.97 \\
0.25 & 0.20 & 0.29 & 0.62 & 0.93 \\
0.30 & 0.16 & 0.25 & 0.58 & 0.89 \\
0.35 & 0.13 & 0.21 & 0.53 & 0.84 \\
0.40 & 0.10 & 0.18 & 0.48 & 0.80 \\
\hline
\end{tabular}


We see that the ratios of the components range from near 2 for small values of Poisson's ratio to about 4 for values of Poisson's ratio near 0.40 . This suggests that the shear source is a better generator of Rayleigh waves since previously we showed that the ratio of the source amplitudes was one.

This conclusion is further supported by a comparison of the energy radiated in the form of Rayleigh waves for the two sources. Using the results of Appendix $C$, we obtain the values given in Table 2.

Table 2. Energy radiated in the form of Rayleigh waves by shear and compressional line source buried in an infinite medium.

\begin{tabular}{lcccc}
\hline $\begin{array}{c}\text { Poisson's } \\
\text { ratic }\end{array}$ & $16 \frac{\mathrm{h}^{2}}{\mathrm{G}} \mathrm{E}_{\mathrm{RC}}$ & $16 \frac{\mathrm{h}^{2}}{\mathrm{G}} \mathrm{E}_{\mathrm{RS}}$ & $\mathrm{E}_{\mathrm{RS}} / \mathrm{E}_{\mathrm{RC}}$ & $\mathrm{V}_{\mathrm{s}}^{2} / \mathrm{V}_{\mathrm{p}}{ }^{2}$ \\
\hline 0.10 & 10.5 & 59.9 & 5.7 & 0.44 \\
0.15 & 8.84 & 59.7 & 6.8 & 0.41 \\
0.20 & 7.31 & 59.6 & 8.3 & 0.38 \\
0.25 & 5.96 & 59.6 & 10.0 & 0.33 \\
0.30 & 4.77 & 59.7 & 12.0 & 0.29 \\
0.35 & 3.76 & 60.1 & 16.0 & 0.23 \\
0.40 & 2.90 & 60.7 & 21.0 & 0.17 \\
\hline
\end{tabular}

$E_{R C}=$ the Rayleigh wave energy per unit length generated by the compressional source.

$\mathrm{E}_{\mathrm{RS}}$ = the Rayleigh wave energy per unit length generateu by the shear source. $\mathrm{G} \quad=$ shear modulus.

$v_{\mathrm{s}}^{2} / v_{\mathrm{p}}^{2}=$ ratio of the body-wave energy radiated by the shear source to the corresponding energy from the compressional source in the same scaled time interval.

From the table we see that the energy contained in the Rayleigh waves generated by the shear source is an order of magnitude greater than the corresponding energy from the compressional source for Poisson's ratios appropriate for earth materials. It also appears that this ratio iacreases as Poisson's ratio increases, However, since the shear energy from an explosion must be generated by some conversion process, e.g., geometry or material inhomogeneity, we have to consider the fact that the explosion is still primarily a source of compressional waves. As we increase Poisson's ratio, the compressional source becomes a more efficient radiator of body waves as is seen from the column $v_{s}^{2} / V_{p}^{2}$ and Appendix B. Thus. from the evasion viewpoint, we must be cautious about ihe advantages to be gained by detonating explosions in media with larger Poisson's ratios.

However for Poisson's ratios appropriate for rocks, we see that on the basis of amplitudes the Rayleigh wave amplitude for the shear source is approxinately three times as large as that for the comparable compressional source. On the basis of 
energy, the shear source is ten to thirty times as strong, depending on whether we normalize the sources on the basis of the residual strain energy or the energy radiated into the far field.

\section{Some Comments on the Generation of Rayieigh Waves}

Although the generation c $i$ higher mode Rayleigh waves in layered media is readily explained by constructive interference (see for example, Tolstoy and Usdin, ${ }^{12}$ ) the physical mechanism by which the fundamental mode is generated is not completely understood. Various authors have presented explanations and/or observations related to this problem (see Mateker, ${ }^{11}$ for example). We shall offer a description of the generation model which incorporates features of both Dix's ${ }^{6}$ and Mateker's ${ }^{11}$ models.

There are several features of the Rayleigh wave which make it particularly interesting. Its period is longer and its velocity slowrr than either the compressional or shear waves which generate it. It does not exist in infinite media or in liquids, Even if there is a boundary in a solid, it cannot be generated without curvature of the wave front or the boundary or at least variation in amplitude along a plane-wave front. Also, the motion is described as lecaying exponentially with distance from the surface so that the energy propagates only parallel to the surface. These latter features are, of course; subject to some qualification since the phenomenon must become established at some point and must then propagate to depth if it arises from a localized source. Another characteristic is that the particle motion at the free surface is very roughly a retrograde ellipse in the case of a localized source. This may suggest that the motion is not due to pure shear or compression and/or may arise from some type of distributed region of energy radiation. Finally, several of the theories of generation take note of the lact that the region over the source exhibits a long-term displacement.

Any attempt to explain these features has to consider the whole process of wave propagation from the scurce to the field point. Therefore we present a heuristic description of the process as we see it. For the sake of concreteness we shall speak in terms of the compressional source and of zones. The latter merely reprasent a region of matter. As the energy radiates (symmetrically) from the source outward and the disiurbance reaches a zone, that zone is distorted, compressed, and begins to move. It attempts to release the internal energy stored in it by radiating energy in all directions. The backward radiation slows the zone behind the wave front. The forward radiation causes the iront to advance by affect: ig the nexi zone. If the wave front is plane, cylindrical, or spherical and of u.iform amplitude, the symmetry is preserved and there is no net energy flux along the wave front but only normal to it. If these conditions are not met, there may be a net tnergy flux along the wave front as well as normal to it and the backward radiation will no longer cause a symmetric decay.

When the disturbance reaches the free surface, the energy cannot propagate acros the boundary and is reflected back into the material. For a zone at the free 
surface directly above the (symmetric) source, the energy is reflected directly back. This zone is not slowed in the same manner as the interior zones were and, in fact., executes motion such that an incident compression returns as a dilatation. As we move away irom the point over the source, the nature of the interaction of the zones at the surface changes and thus the nature of their motion changes. This change is due to the finite propagation velocity, the change in the angle of incidence, and the fact that the amplitude of the disturbance emanating from the localized source decays with distance due to geometrical spreading. A sketch of several zones and the forces acting on them is shown in Fig. 4.

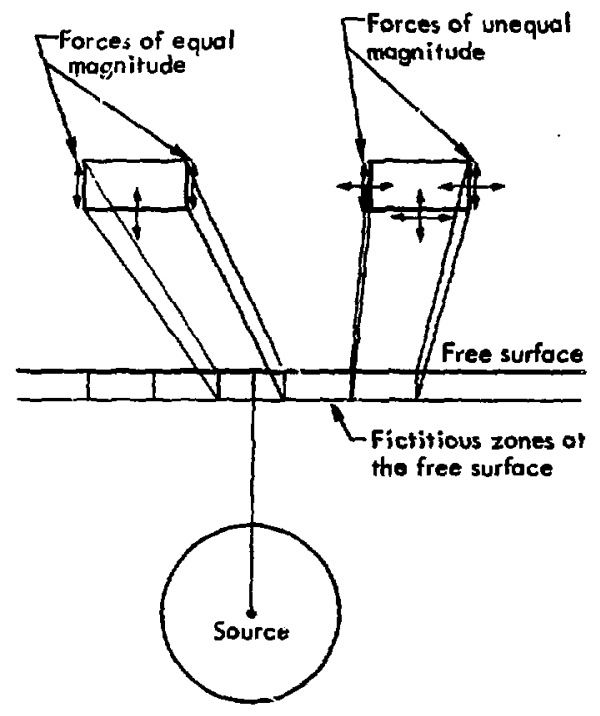

Figure 4. Variation in the forces acting on various zones at the free surface.

The additional asymmetric forces acting on the zones which are off of the axis of symmetry cause these zones to rotate. The presence of this $x$ - ation is related to the generation of shea: waves.

As the reflected waves propagate back through the medium, they differ in both magnitude and direction from the wave which propagated through the region as the direct wave. Thus the rezidual strain fields interact in varying ways, causing a consequent variation in the kinetic and residual internal energies as a function of position. The medium then attempts to readjust itself so as to have the minimum interual energy consistent with the source and boundary constraints. It appears that the reajjustment 
of the residual energy remaining after the passage of the wave fronts gives rise to the Rayleigh waves.

One of the chief sources of the energy imbalance appears to arise from the concentration of energy above the source region. Here the geometrical spreading for the wave striking the free surface is smallest, and thus an initially symmetric wave will have the greatest strength at the free burface at this point, Another source of imbalance in the energy distribution is the variation in the partition of energy between the reflected shear and compressional waves as a function of angle of incidence (see, for example, pp. 30 and 31 in Ref. 3). This partitioning causes an energy flow along the wave fronts of these two waves. The result of these energy gradients is that we have asymmetric radiation of energy back toward the surface as well as in the direction of the advancing wave fronts. We propose that the Rayleigh wave is established as the result of distortional energy flowing out of the region over the source interacting with distortional energy related to the incident and reflected compressional waves flowing back from the advancing shear wave. At the free surface, this energy is also reflected in the form of compressional and distortional energy which then spreads out into the medium and interacts with the incoming energy at late times.

In support of this proposal, we present some figures showing the distributions of various types of energy at four different times as computed from the expressions for the displacements for the compressional source given in Eq. (1). Before discussing these figures in detail, we shall provide some background related to the energy partitioning used.

In Appendix $D$ we have obtained the forms used in computing the energy plots in this report. Following Fung, ${ }^{13}$ we partitioned the energy into three parts: kinetic energy, compressional energy, and distortional energy. The compressional energy is related to volume changes of zones, while the distortional energy is related to changes in shape of the zones. The compressional energy is written in terms of the first strain invariant, whereas the distortional energy is expressed in terms of the de-riatoric strains. Compressional waves in general cause both a change in volume an' a change in shape, and thus contain both compressional and distortional energy. He shear waves have no volume change associated with them, and thus have only disto tional enelgy.

Figure 5 shows the way in which energy is plotted as a function of position in the isometric plots shown in Figs. 6-9. These figures represent oblique views of an orthogonal coordinate systeris which depicts energy density, $E$, as a function of position, $\xi, \zeta$. The surface $E=E(\xi, \zeta)$ is depicted by plotting the curves $E=E(\xi, \zeta)$ for various $\xi_{i^{*}}$ Figures 6 and 7 correspond to the region $0.02 \leq \xi \leq 2.00,0 \leq \xi \leq 0.98$, shown in Fig. 5 as bounded by the solid lines. Figure 8 corresponds to the region $1.02 \leq \xi \leq 3.00,0 \leq \zeta \leq 0.98$, shown in Fig. 5 as bounded by the dashed lines. Figure 9 corresponds to the region $3.02 \leq \xi \leq 5.00,0 \leq \xi \leq 0.98$. This region is not shown in Fig. 5. 


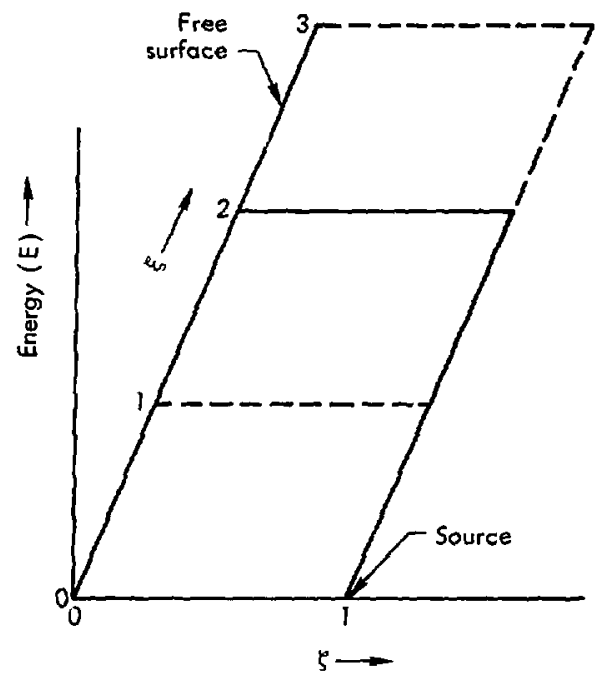

Figure 5. Reference figure for inierpreting the isometric plots.
Although Eq. (1) could be differentiated with respect to space and time in order to obtain the energies, for convenience the derivatives were calculated numerically. Since at the onset of the waves the amplitudes become infinite, the numerical differentiation scheme introduces a considerable amount of noise in the neighborhood of the wave fronts. This phenomenon is especially severe in the compressional energy plots where it introduces noise at the position of the shear wave even though the shear wave has no compressional energy. Although this feature is undesirable, the areas of interest are, for the most part, removed from the noise, and thus we are able to use this approach rather than the more lengthy calculation of the appropriate analytic expressions.

Figure 6 shows the various energies for a solid with Poisson's ratio $=0.25$ at a time when the direct compressional wave has propagated a distance of 2.2 times the depth of burial. The region shown corresponds to the region from the source to the surface in the $\zeta$ direction and fr:m the epicenter to a distance equal to twice the depth of burial in the $\xi$ direction. The source is located in the lower right-hand corner, and the free surface is on the left inclined edge. Although the kinetic energy is relatively ismooth, the extreme variability in the residual strain energy behind the wave front is quite apparent. The major leatures are, of course, the wave fronts. However, we note that there is a considerable amount of energy stored in the epicentral region, and that the kinetic energy is small in the same region. This corresponds to the phenomenon described by Mateker. ${ }^{11}$

Further, we note that the compressional energy has a rather large low (indicated by the arrow), while the distortional energy has a steep gradient at the same point. This region is significant since the Rayleigh wave should appear there. Various other features corresponding to observed and predicted phenomena can be observed.

Figure 7 shows a similar set of plots at a time when the direct compressional wave has propagated a distance equal to 2.5 times the depth of burial of the source. Here we see that the region of low compressional energy has spread, but in addition a region of increasing compressional energy has appeared in the center of this region at the surface. The kinetic energy shows a minimum in the same area. The distortional energy variation is more complex. Although there appears to be an overall decrease 


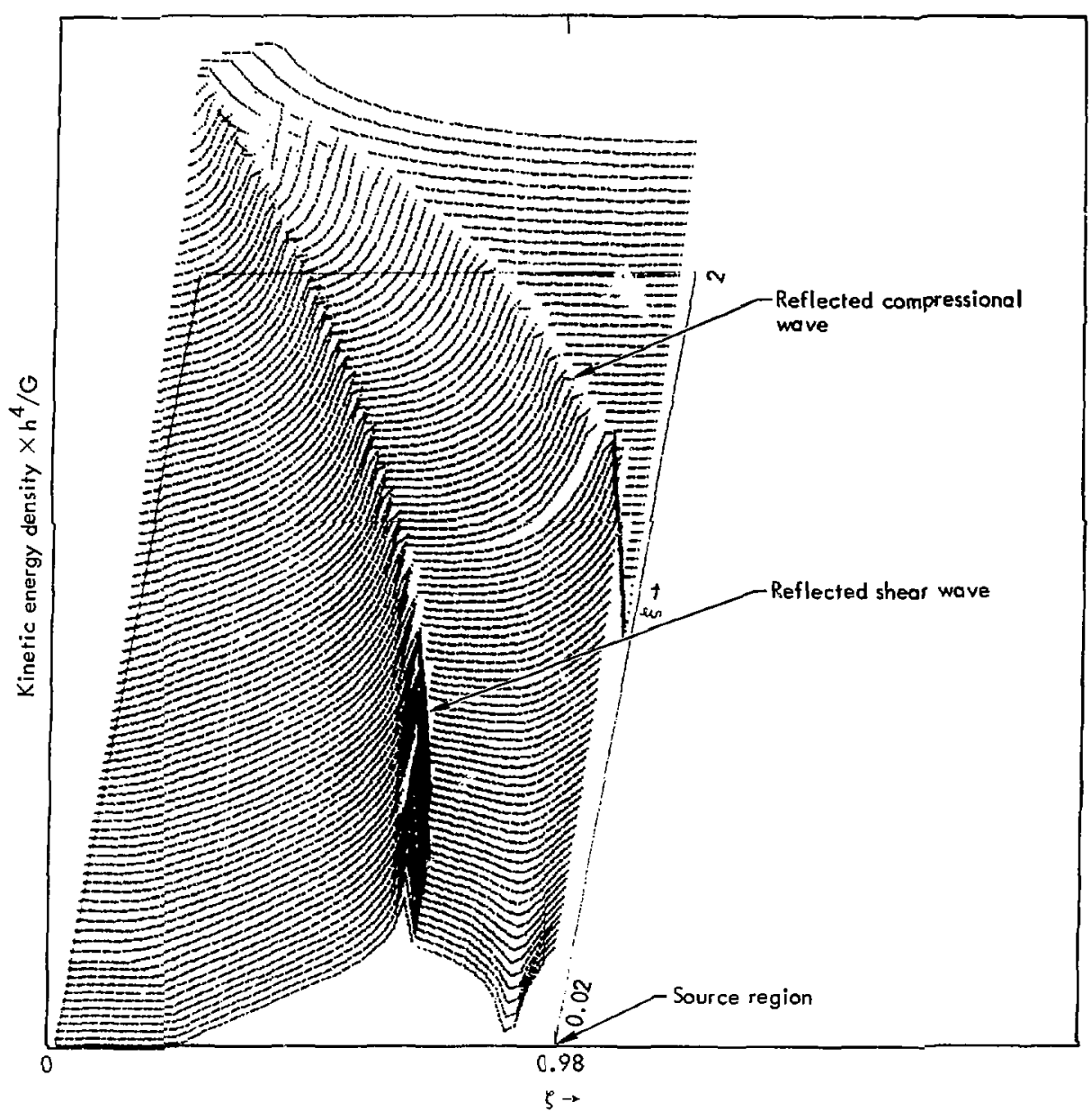

(a) Kinetic energy

Figure 6. Isometric plots of the energy density as a function of position for a compressional source in a solid with Poisson's ratio equal to 0.25 at $\eta=2.2$, $0.02<\xi<2.0,0<\xi<0.98$. 


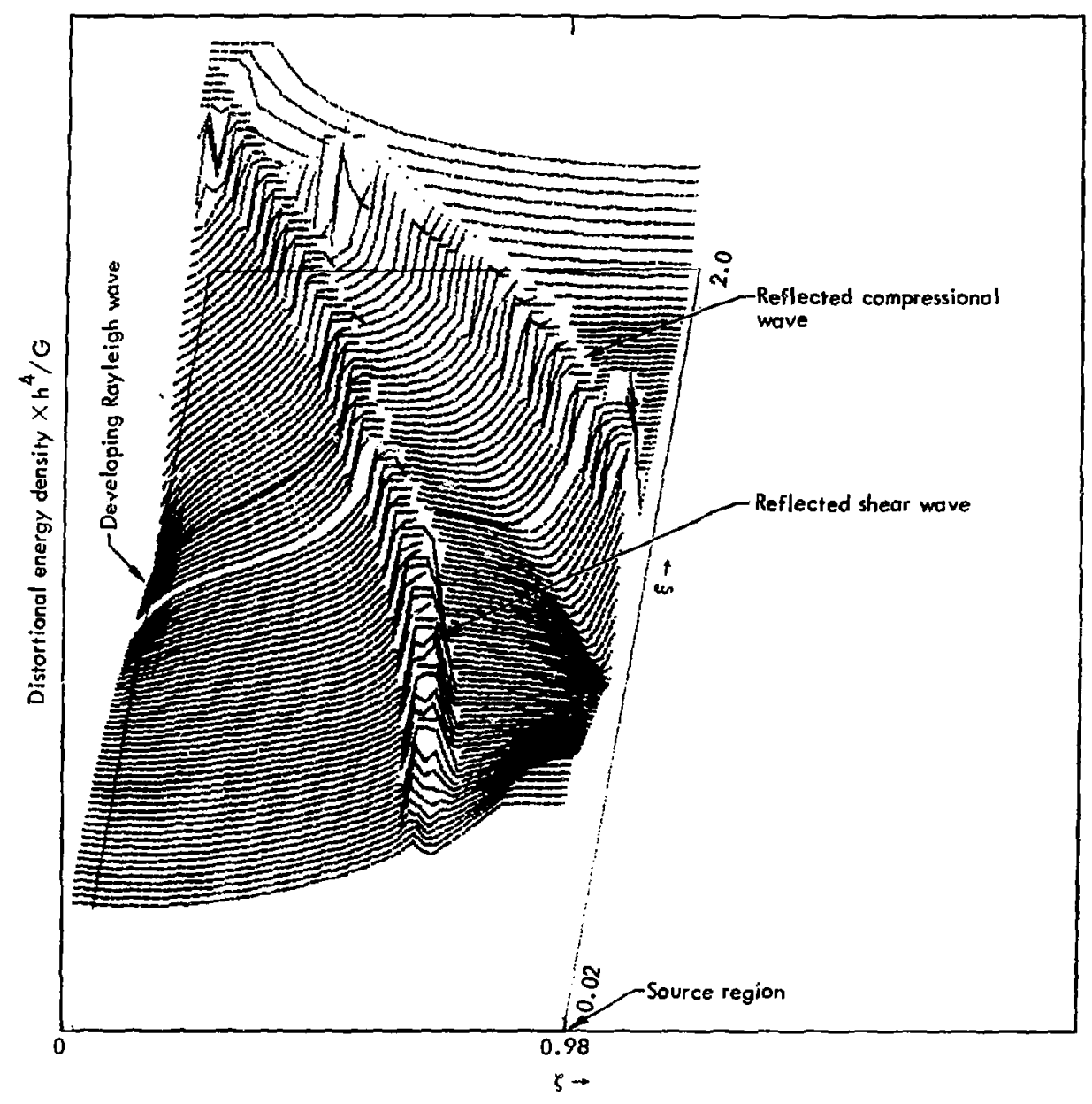

(b) Distortional energy

Figure 6 (contd.) 


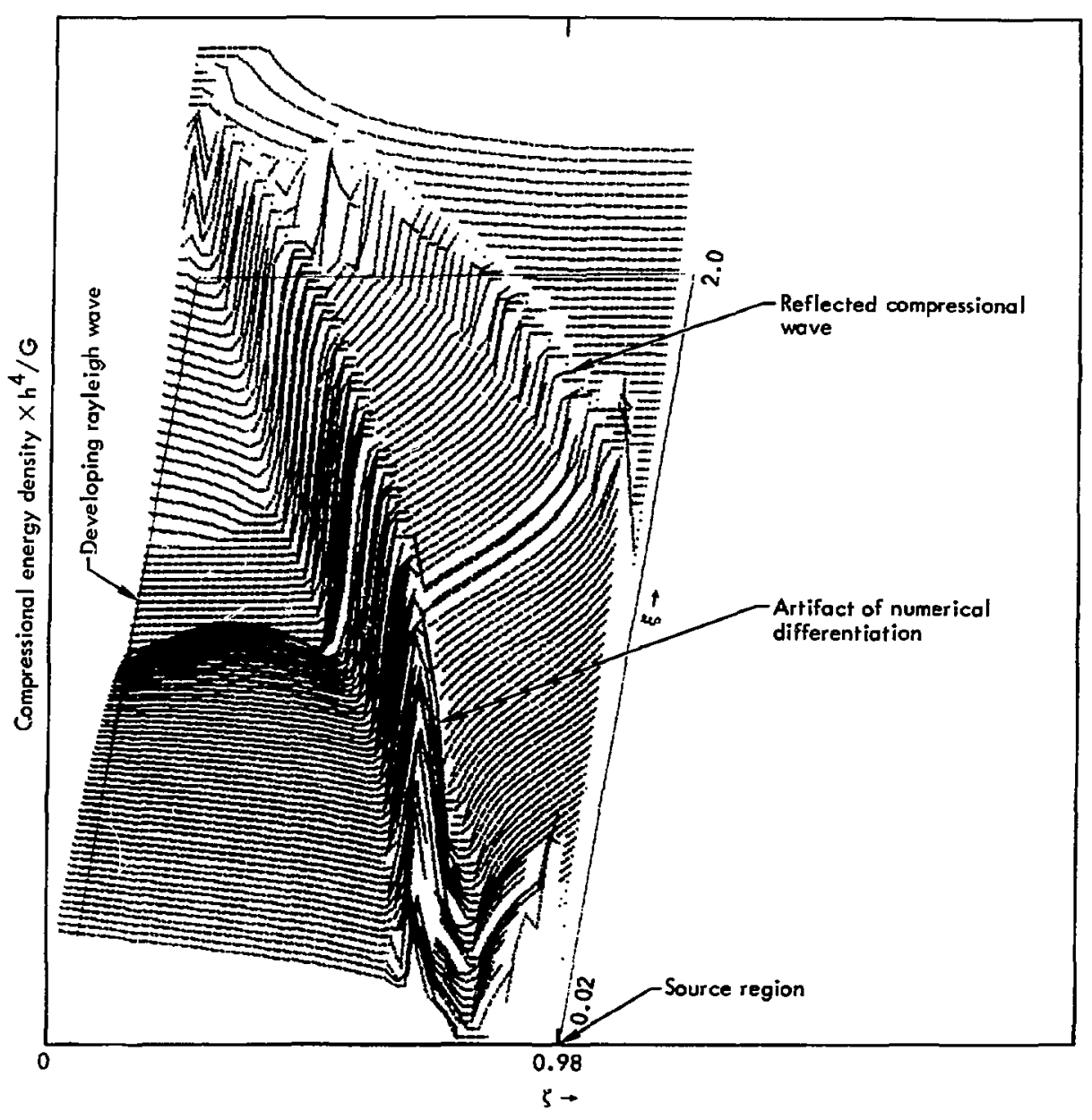

(c) Compressional energy

Figure 6 (contd.) 


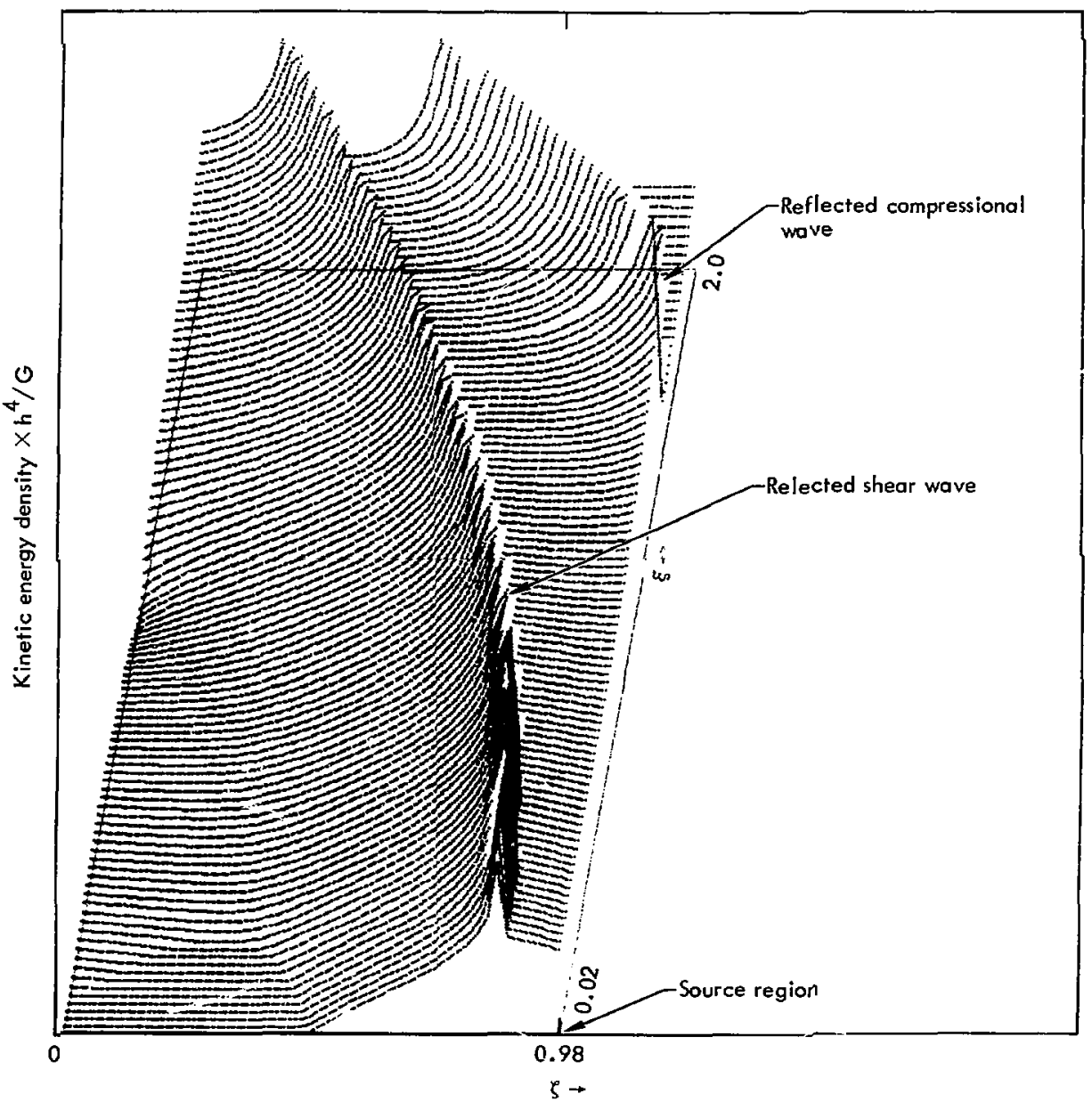

(a) Kinetic energy

Figure 7. Isometric plots of the energy density as a function of position for a compressional source in a solid with Poisson's ratio equal to 0.25 at $\eta=2.5$, $0.02<\xi<2,0,0<\xi<0.98$. 


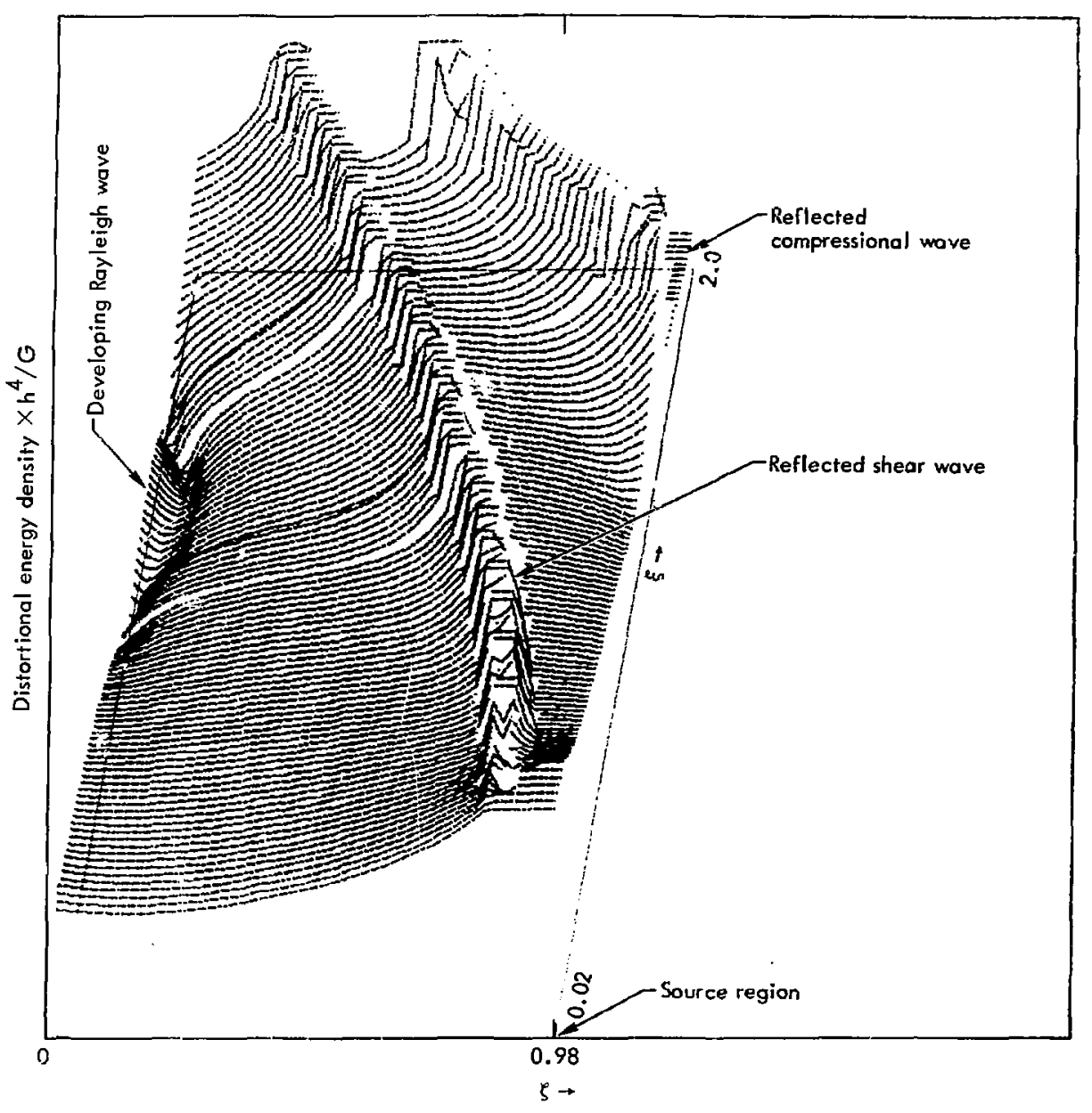

(b) Distortional energy

Figure 7 (contd.) 


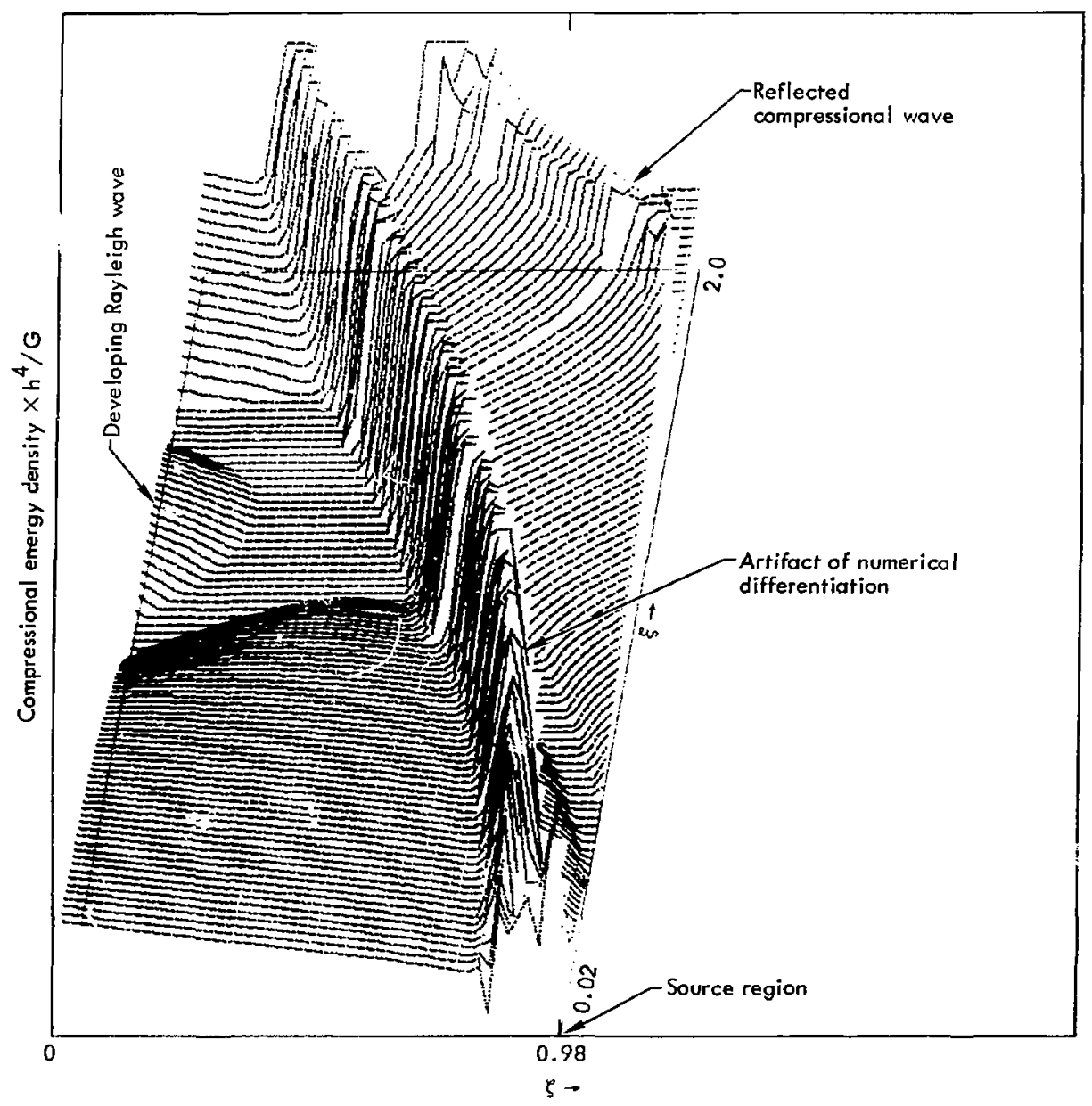

(c) Compressional energy

Figure 7 (contd.) 


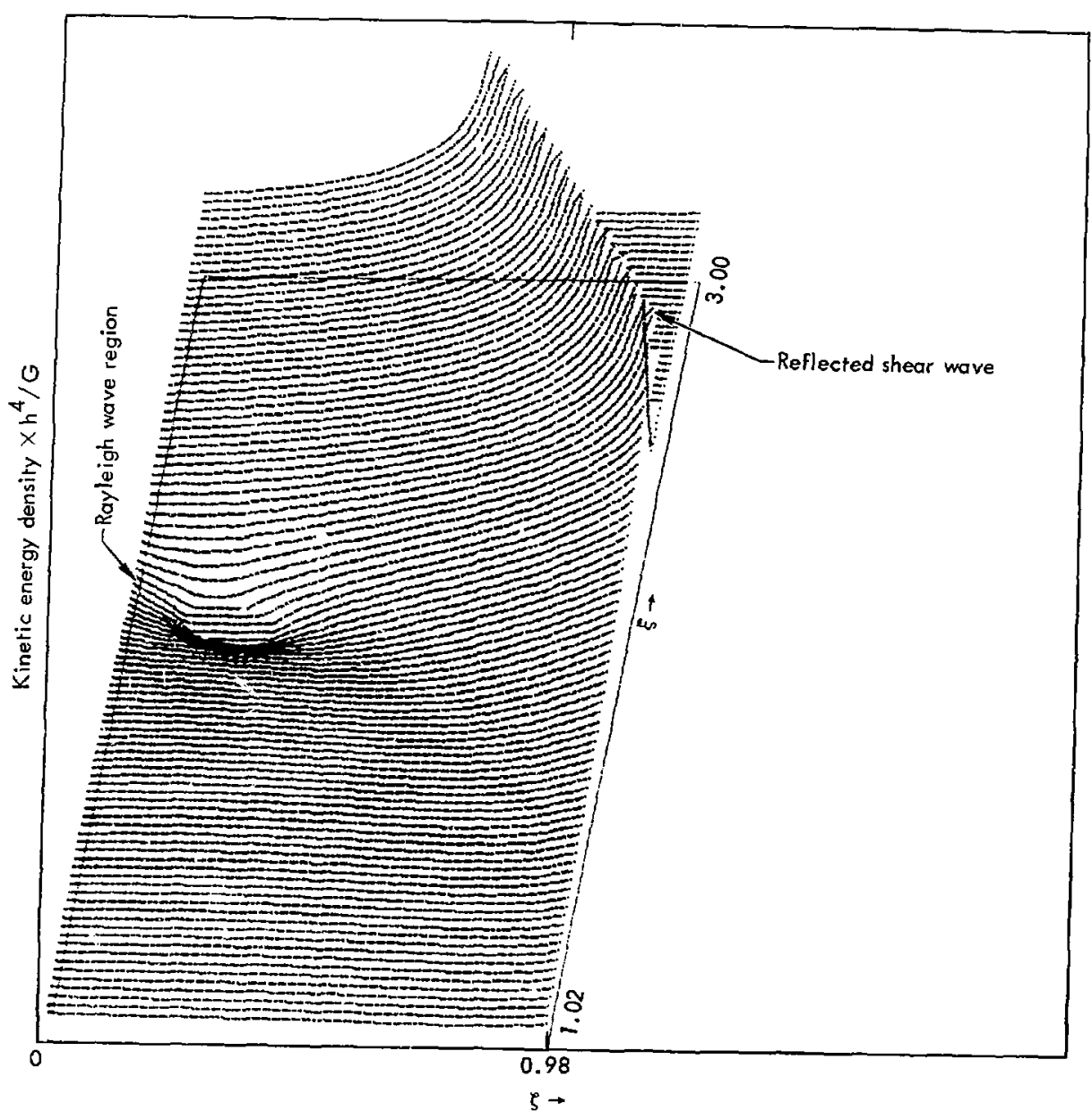

(a) Kinetic energy

Figure 8. Isometric plots of the energy density as a function of position for a compressional source in a solid with Poisson's ratio equal to 0.25 at $\eta=4.0$. $1.02<\xi<3.00,0<\zeta<0.98$. 


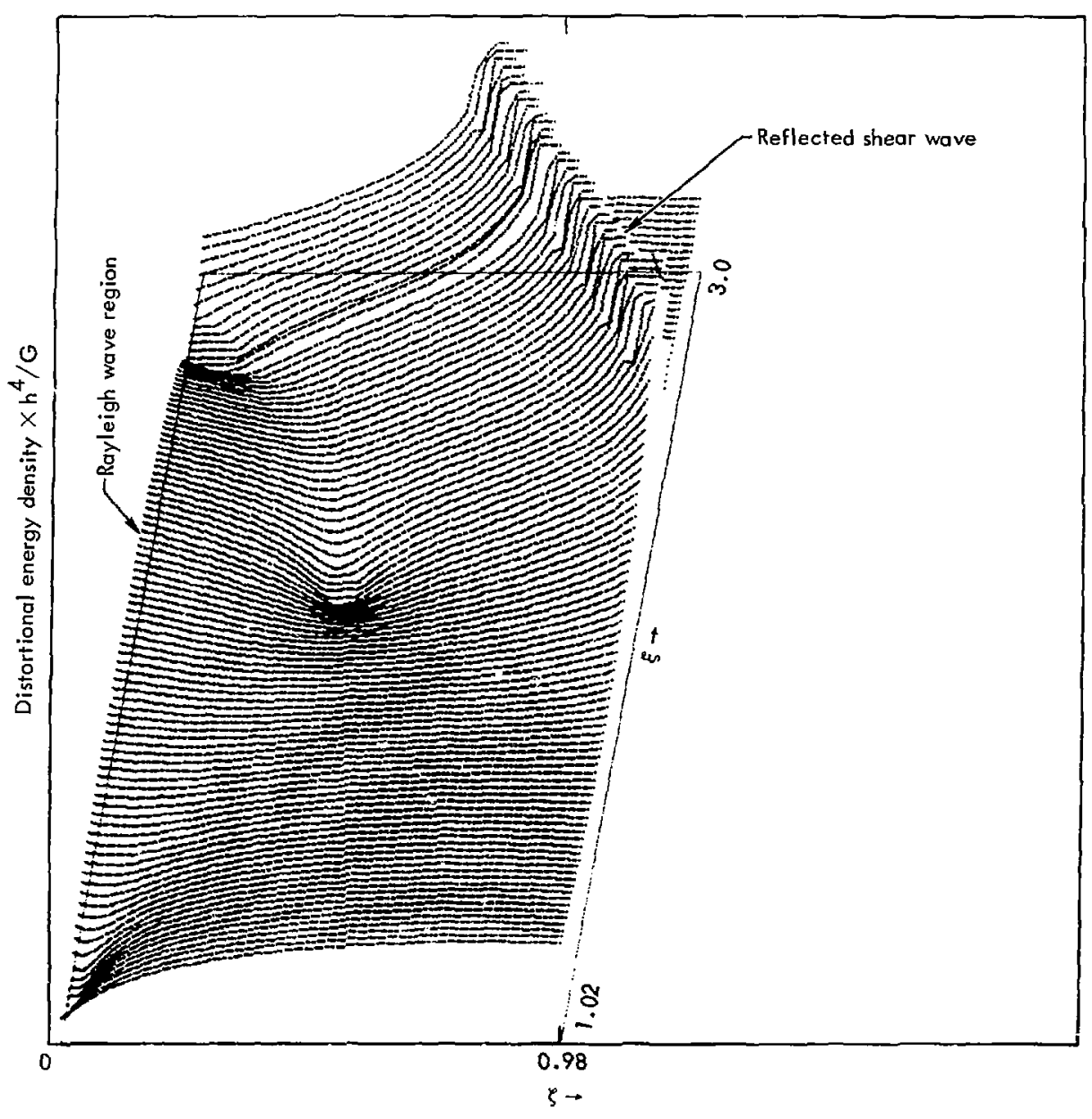

(b) Distortional energy

Figure 8 (contd.) 


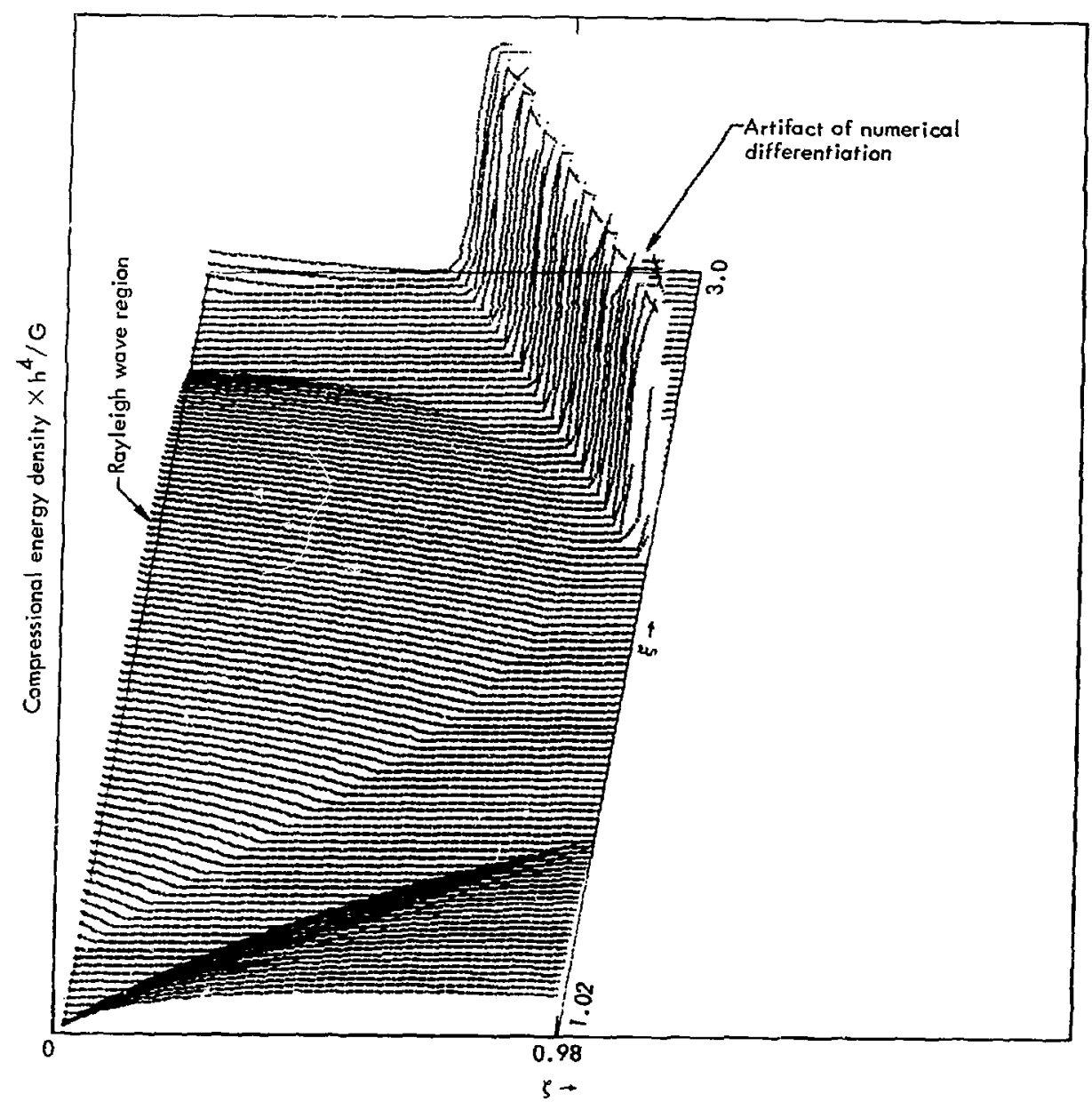

(c) Compressional energy

Figure 8 (contd.) 
in energy in this regiun, there is ar increase near the surface. These plots show the initiation of the Rayleigh wave.

An explanation which is consistent with these plcts is that disiorticnal energy from the epicentral region and the shear-wave residual radiates into this region. Upon striking the free surface, it is both reflected and converted into compressional energy. This description differs from previous ones in that it takes into account both the source region anc the effects of the variation in energy density due to the variation in reflection coefficients as a function of angle. It is postulated that it is this latter effect that accounis for the backward radiation from the outgoing waves.

Figure 8 shows energy plots for the region $1.0 \&<\xi<3.00,0<\xi<0.98$ at a time $\eta=4.0$. Here we see that the converted compressional energy has spread out and advanced. The kinetic energy has a low in it, while the distortional energy has a pattern of three lows. These features are the result of outward radiaticn from the source region and backward radiation from the shear wave. The reflection of these energies at the free surface and their conversion into compressional energy forms the initial phase of what will be a stable propagating interference pattern.

Figure 9 shows the form of the propagating patterns with elongate lows in the kinetic energy and the distortional energy which slightly overlap. Ti ase are sur rounded by regions with relatively steep gradients. The compressional energy appears to be relatively smocth. This pattern persists at large distances. Thus we see that inhomogeneities in the kinetic and distortional energy distributions appear to play the greatest role in the propagation of Rayleigh waves once it is established.

\section{Conclusions}

From the examination $f$ the displacements and energies irom buried compressional and line sources, we conclude that:

1. A localized shear source is a more efficient generator of Rayleigh waves than is a compressional source of the same strength. Although the discontinuities and the differences in time scales involved in the particular solutions used render an exact comparison diffieult, if we compare the two sources in terms of scaled time, we find that: the amplitudes and residuai strain energies are tr $₹$ same: the compressional source radiates more energy per unit length by a factor or $v_{p}{ }^{2} / v_{s}{ }^{2}$; and the Rayleigh wave energy per unit from the shear source is an order of magnitude greater than that from the compressional source for Poisson's ratios $\div 0.25$. For Poisson's ratios near 0.25, the shear source generates Rayleigh waves with amplitudes thrce times those of the compressional source. Thus the energy contained in the Rayleigh waves from the shear source if 10 to 30 times that of the compressional source, depending on whether the residual strain energy or the radiated energy is used as the basis of normalization.

2. The wauduism for generating Rayleigh waves involves an interaction between the residual distortional energy related to the comprossional waves flowing out from the 


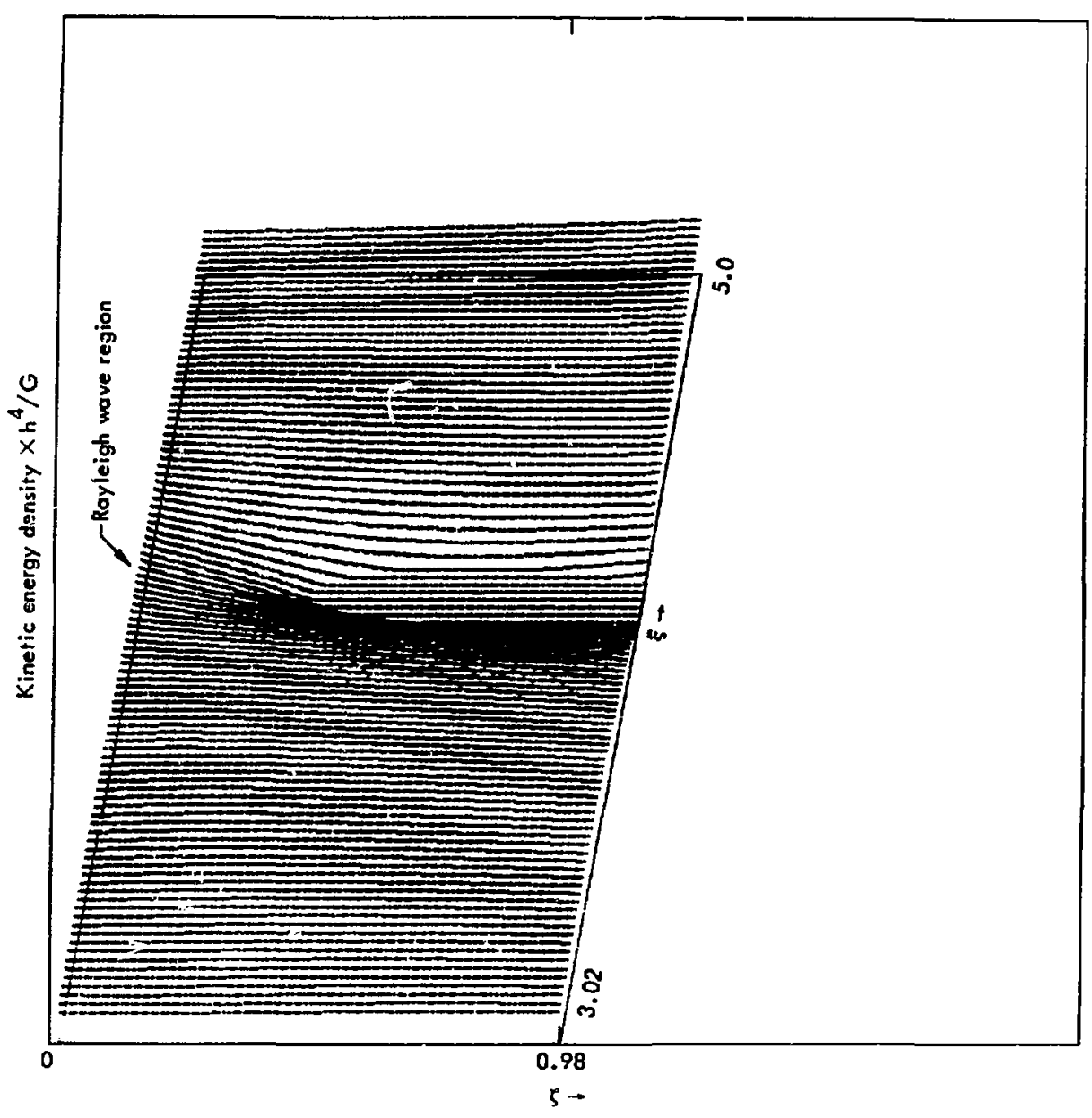

(a) Kinetic energy

Figure 9. Isometric plots of the energy density as a function of position for a compressional source in a solid with Poisson's ratio equal to 0.25 at $\eta=7.8$, $3.02 \leq \xi \leq 5.00,0 \leq \zeta \leq 0.98$. 


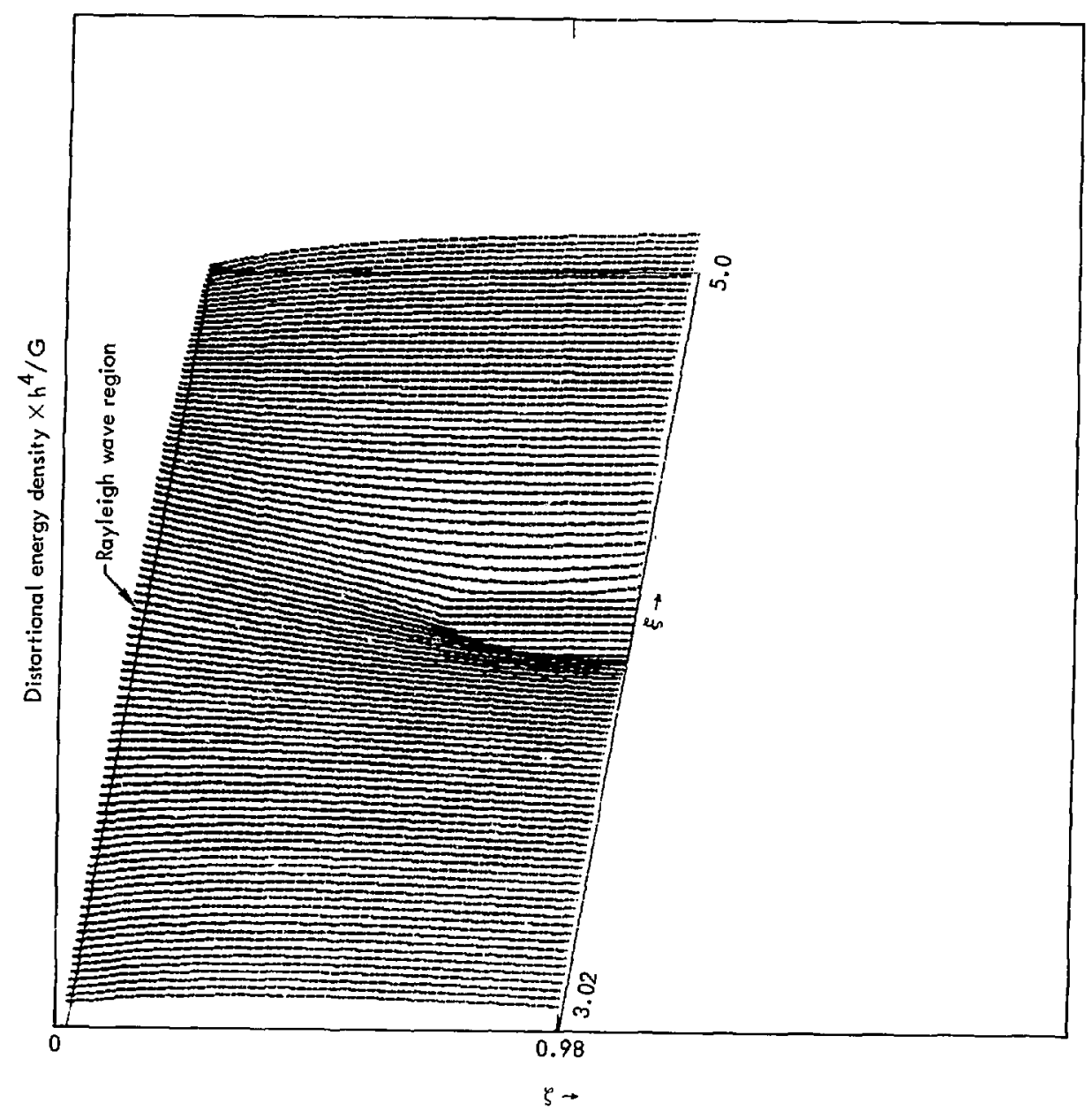

(b) Distortional energy

I sure 9 (contd.) 


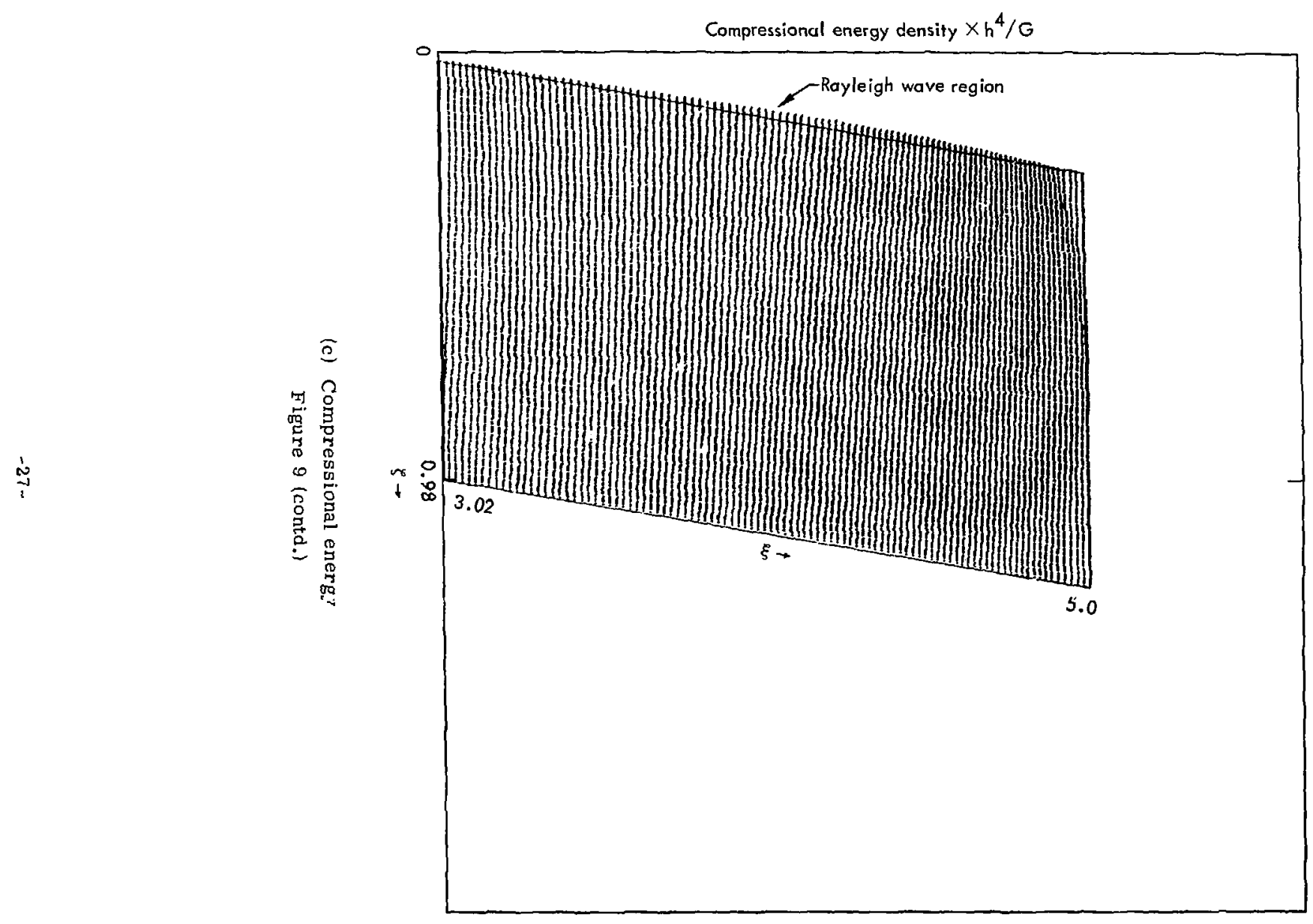


source region and radiation from the residual distortional energy remaining after the passage of the shear wave. This energy distribution is not uniform because the reflected shear wave varies in amplitude along its wave front, and the wave front is not cylindrical. (The latter statement is true for a compressional source. For a shear source, the reflected shear wave has a cylindrical front and has only a variation in amplitude.) The radiation from these two source regions not only interact with each other, but also with the free surface. This causes a conversion from distortional to reflected compressional energy. This triple interaction establishes a stable energy pattern which then propagates along the surface.

3. The combined effect of conclusions 1 and 2 is to suggest that if we can increase the distortional energy above the suurce and in the reflected shear wave from large angles of incidence (say $>45^{\circ}$ ), we can increase the Rayleigh waves. A possible way to accomplish the latter suggestion is to use shaped cavities, possibly in the form of ellipsoids, to both generate distortional energy and radiate energy asymmetrically. If either approach is practical, it appears that the surface-wave-body-wave magnitude discriminate can be compromised at will.

\section{Acknowledgments}

It is a pleasure to thank Howard $C$. Rodean for his encouragement and constructive suggestions and James A. Viecelli for many profitable discussions. My initial interest in the generation of Rayleigh waves was stimulated during the course of mary rewarding exchanges with Emil J. Mateker (now with Western Geophysical Co.) when we worked together at both Washington and St. Louis Universities. This work was partially funded by the Advanced Research Project Agency. 


\section{Appendix A \\ The Derivation of the Solution for a Finite Source of Shear Energy Buried in a Homogeneous Elastic Half Space}

We shall consider a line source of shear waves buried at a septh below the free surface of a semi-infinite homogeneous elastic medium (see Fig. 1). Garvin ${ }^{4}$ and Nagumo ${ }^{9}$ among others have carried out similar developments for the displacements at the free surface for both shear and compressional sources and at all interior points for the case or a compressional line source. For the sake of completeness, we shall outline the solution for the shear line source using techniques similar to theirs.

The displacement vector $\vec{s}$ satisfies the equation of motion

$$
(\lambda+2 G) \nabla(\nabla \cdot \vec{s})-G \nabla \times(\nabla \times \vec{s})=p \frac{\partial^{2} \vec{s}}{\partial t^{2}} \text {. }
$$

where

$\lambda$ and $G$ are Lame's constants and

$p$ is the density of the medium.

We introduce the dispiacement potentials and $\vec{A}$

where

$$
\text { we choose } \nabla \cdot \vec{A}=0 \text {. }
$$

The potentials $\phi$ and $\vec{A}$ satisfy the scalar and vector wave equations respectively, i. e..

$$
\nabla_{\phi}=\frac{1}{v_{c}^{2}} \frac{\partial^{2} \phi}{\partial t^{2}}
$$

and

$$
\nabla^{2} \vec{A}=\frac{1}{V_{s}^{2}} \frac{\partial^{2} \vec{A}}{\partial t^{2}}
$$

where

$$
\begin{aligned}
& v_{c}=\sqrt{\frac{\lambda+2 G}{\rho}}=\text { the compressional wave velocity, and } \\
& v_{S}=\sqrt{\frac{G}{\rho}}=\text { the shear wave velocity. }
\end{aligned}
$$

If we o.fine the Laplace transform in the usual way. 


$$
\bar{f}(x, y, p)=\int_{0}^{\infty} f(x, y, t) e^{-p t} d t
$$

and consider all terms with the bars above them to be transformed fuantities, the transforms of Eqs. $(A-3)$ and $(A-4)$ are given by:

$$
\nabla^{2}-\frac{p^{2}}{v_{c}^{2}} \bar{\phi}
$$

and

$$
\nabla^{2} \overrightarrow{\vec{A}}=\frac{p^{2}}{V_{S}^{2}} \quad \overrightarrow{\vec{A}}
$$

We can represent the solution to the shear source problem as

$$
\begin{aligned}
& \bar{\phi}=\int_{0}^{\infty} B(k) e^{-y n_{c}} \sin k x d k \\
& \vec{A}=\bar{A}_{z} \vec{e}_{z}
\end{aligned}
$$

with

$$
\bar{A}_{z}=\bar{A}_{0}(x, y, p)+\int_{0}^{\infty} c(k) e^{-y \eta_{s}} \cos k x d k
$$

where

$$
\begin{aligned}
& n_{c}=\sqrt{k^{2}+\frac{p^{2}}{v_{c}^{2}}}, \operatorname{Re} n_{c}>0: \\
& n_{s}=\sqrt{k^{2}+\frac{p^{2}}{v_{s}^{2}}}, \text { Re } \eta_{s}=0 ; \\
& \vec{e}_{z}=\text { unit vector in the }+z \text { direction. }
\end{aligned}
$$

and $\mathrm{B}(\mathrm{k})$ and $\mathrm{C}(\mathrm{k})$ are to be adjusted to meet the boundary conditions related to the vanishing of stress at the iree surface. The above forms are the analogues of the forms chosen by Garvin for the compressional wave source. 
A key point in Garvin's original problem was his recognition of the form of the function corresponding to $\bar{A}_{0}(x, y, k)$ such that inversion was possible. Using his results as a guide, we chose

$$
\bar{A}_{0}(x, y, p)=\frac{a}{2 p} K_{0}\left(\frac{p r}{V_{c}}\right)=\frac{a}{2 \pi} \int_{0}^{\infty} e^{-|y-h| \eta s} \frac{\cos k x}{\eta_{s}}
$$

where $K_{0}$ is the modified Bessel function of the serond kind and pero order.

$$
r_{-}=\sqrt{x^{2}+(y-h)^{2}} \text {. }
$$

and

a is an arbitrary constant.

The inverse Laplace transform of $\bar{A}_{0}(x, y, p)$ is given by

$$
A_{0}(x, y, t)=\frac{a}{2} \cosh ^{-1}\left(\frac{V_{g} t}{r_{-}}\right) H\left(t-\frac{r_{-}}{V_{s}}\right)
$$

where

$$
H\left(t-\frac{r_{-}}{V_{s}}\right)
$$

is the unit step function defined previously.

The displacements corresponding to the source function are given by:

$$
\vec{s}=s_{x} \vec{e}_{x}+s_{y} \vec{e}_{y}
$$

with

$$
s_{x}=\frac{\partial A_{0}}{\partial y}=-\frac{(y-h)}{r_{-}} \frac{a t}{2 r_{-} \sqrt{t^{2}-\left(\frac{r-}{V_{s}}\right)^{2}}} \text { for } t>\frac{r_{-}}{V_{s}}
$$

and

$$
s_{y}=-\frac{\partial A_{0}}{\partial x}=\frac{x}{r_{-}} \frac{a t}{2 r_{-} \sqrt{t^{2}-\left(\frac{r_{-}}{V_{s}}\right)^{2}}} \quad \text { for } t>\frac{r_{0}}{V_{s}} \text {. }
$$

and

$$
\vec{e}_{x^{\prime}}, \vec{e}_{y}=\text { unit vectors in the }+x \text { and }+y \text { directions respectively. }
$$




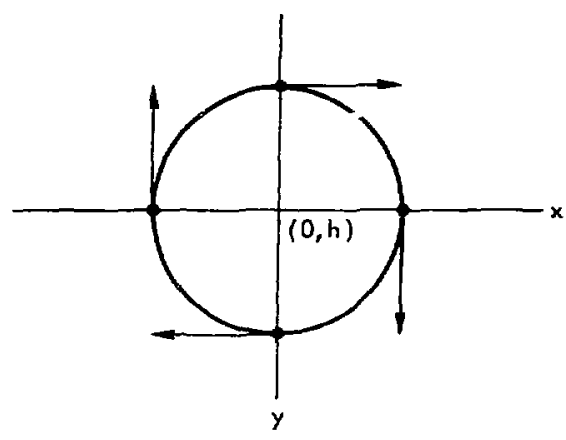

Ficure A-1. Directions of motion of selected points about the line source.
Thus we see that this source of shear waves emits waves whose displacement history is shown in Fig. 2 of the text. The displacements of the source are shown in rigure $A-I$. From the figures we see that the displacements correspond to a sudden clockwise rotation about the source axis, followed by a gradual incomplete recovery. If we substitute the forms $\overrightarrow{\bar{A}}$ and $\bar{\phi}$ into Eq. $(A-2)$, 've have a representation of the transformed displacement which has to satisly the transformed boundary conditions representing the vanishing of the stress at the free surface, i.e.,

$$
\left.\overline{\mathrm{T}}_{y y}\right|_{y=0}=0=\rho\left(v_{c}^{2}-2 v_{s}^{2}\right) \frac{\partial \tilde{s}_{x}}{\partial x}+\rho v_{c}^{2} \frac{\partial \bar{s}_{y}}{\partial y}
$$

and

$$
\left.\overline{\mathrm{T}}_{x y}\right|_{y=0}=0=G\left(\frac{\partial \bar{s} x}{\partial \bar{y}}+\frac{\partial \bar{s} y}{\partial x}\right) .
$$

Making the appropriate substitutions and solving for $B(k)$ and $C(k)$, we find that

$$
B(k)=\frac{2 a k}{p} v_{s}^{2} \frac{\left(p^{2}+2 k^{2} v_{s}^{2}\right)}{\Delta\left(k^{2}\right)} e^{-h \eta_{s}}
$$

and

$$
C(k)=-\left[\frac{a}{2 p \eta_{s}}+\frac{4 a}{p} \frac{k^{2} \eta_{c} v_{s}^{4}}{\Delta\left(k^{2} !\right.}\right] e^{-h \eta_{s}} .
$$

where

$$
\Delta\left(k^{2}\right)=\left(p^{2}+2 v_{s}^{2} k^{2}\right)^{2}-4 k^{2} v_{s}^{4} n_{s} n_{c}
$$

If we substitute these forms into Eqs. $(A-7)$ and $(A-8)$ and then use the integral representation of Eq. (A-11), we: may write an integral representation of the transiormed components of displacement described in Fq. (A-2). if we use the change of variable $u=k v_{s} p$, we may write 
$\bar{s}_{x}=\operatorname{Re} \frac{a}{2 V_{s}} \int_{0}^{\infty}\left\{[\operatorname{sgn}(h-y)] e^{-p \frac{|y-h|}{V_{s}} \sqrt{1+u^{2}}}+e^{-p(y+h)} \frac{\sqrt{1+u^{2}}}{V_{s}}\right.$

$$
\begin{gathered}
+^{8} \frac{u^{2} b \sqrt{1+u^{2} s^{2}}}{\Delta_{s}\left(b, u^{2}\right)} \frac{\sqrt{1+u^{2}}}{e_{s}} e^{\frac{-p(h+y)}{v_{s}} \sqrt{1+u^{2}}} \\
-4 u^{2} \frac{\left(1+2 u^{2}\right)}{\Delta_{s}\left(b, u^{2}\right)} e^{-\frac{p h}{v} \sqrt{1+u^{2}}-\frac{p y}{V_{c}} \sqrt{i+\frac{u^{2}}{b^{2}}}} e^{-i \frac{p u x}{V_{s}}} d u
\end{gathered}
$$

and

$$
\begin{gathered}
\bar{s}_{y}=-\operatorname{Im} \frac{a}{2 V_{s}} \int_{0}^{\infty} \frac{u}{\sqrt{1+u^{2}}} e^{\frac{-p|h-y|}{V_{s}} \sqrt{1+u^{2}}}-\frac{u}{\sqrt{1+u^{2}}} e^{-\frac{p(h+y)}{V_{s}} \sqrt{1+u^{2}}} \\
-\frac{8 b u^{3} \sqrt{1+\frac{u^{2}}{b^{2}}}}{-\frac{-p(h+y)}{v_{s}\left(b, u^{2}\right)}} e^{\frac{v_{1+u^{2}}}{s}}
\end{gathered}
$$

$$
\left.+\frac{4 b u \sqrt{1+\frac{u^{2}}{b^{2}}}}{\Delta_{s}\left(b, u^{2}\right)}: a+2 u^{2}\right) e^{-p \frac{y}{p} \sqrt{1+u^{2} b^{2}}-p \frac{h}{V_{s}} \sqrt{1+u^{2}}}
$$

$$
x e^{-i \frac{p u x}{V}} \text { du }
$$

where

$$
\begin{aligned}
\operatorname{sgn}(h-y) & =+1 h>y \\
& =-1 h<y, \\
b & =\frac{v_{s}}{V_{p}} .
\end{aligned}
$$

Re and Im reter to the real and imaginary parts,

$$
\Delta_{s}\left(b, u^{2}\right)=\left(1+2 u^{2}\right)^{2}=4 b u^{2} \sqrt{1+u^{2}} \sqrt{1+u^{2} b^{2}} \text {. }
$$


The various terms in the expressions for the two components can be interpreted as follows: The first term represents the contribution of the source; the second term represents the contribution of an image located at $(0,-h)$; the third term is a correction to the image term to allow for the energy lost from an ideal image because of the partial conversion from shear to compressional waves at the free surface; and the fourth term represents that part of the disturbance which is related to the compressional wave arising at the frece surface.

The inverse Laplace transforms of the first three terms zan easily be obtained by examining similar integrals in Garvin's paper. The inverse of the last term can be obtained in the same way that Nagumo evaluated the converted wave from the compressional source.

We outline the approach below by considering the inversion of the fourth term in $\mathbf{s}$

We might summarize our objective at this point as follows: We want to modify the form of the transformed term under consideration so that it takes the form

$$
T(p)=\int_{0}^{\infty} e^{-p t} F(t) d t
$$

Then, since

$$
\bar{T}(p)=\int_{0}^{\infty} e^{-p t} f(t) d t .
$$

We reccgnize that $F(t)=f(t)$ is the time runction corresponding to thr Laplace transform $\bar{r}(p)$, With this goal in mind, we introduce a change of variable,

$$
t=\frac{i u_{4} x}{v_{s}}+\frac{y}{v_{s}} \sqrt{b^{2}+u_{4}^{2}}+\frac{h}{v_{s}} \sqrt{1+u_{4}^{2}} .
$$

Thus we have for the fourth term in $s_{x}$ (let us call it $s_{x 4}$ ),

$$
\begin{aligned}
s_{x 4} & =-R e \frac{2 a}{v_{s}} \int_{0}^{\infty} u_{4}^{2}\left(1+2 u_{4}^{2}\right) e^{-\frac{p h}{v_{s}} \sqrt{1+u_{4}^{2}}-\frac{p y}{v_{c}} \sqrt{1+u_{4}^{2} b^{2}}-\frac{i \rho u_{4} x}{v_{s}}} d u \\
& =-\operatorname{Rc} \frac{2 a}{v_{s}} \int_{c}\left[u_{4}(t)\right\}^{2}\left\{1+2\left(u_{4}(t)\right\}^{2}\right\} e^{-p t} \frac{d u_{4}(t)}{d t} d t
\end{aligned}
$$

\footnotetext{
Note: doftoof ${ }^{14}$ has developed a generalliced method for solving the same types of problems. The gresent approach is sufficient for our purposes here.
} 
where

$u_{4}(t)$ represents $u_{4}$ as a function of $t$ as derived (rom Eq. (A-24),

and $c$ is the contour in the complex $t$ plane that corresponds to the real axis from 0 to so in the $u$ plane.

In order to determine $u_{4}$ as an expicit function of $t$, we would have to solve a 4 thdegree equation which arises when the radicals ane removed. Although this can be clone explicitly, the expressions obtained are not illuminating. We take the position that since the t:se of the final results $w$ thl depend upon computing the yalues of the displacements at various points, it is sufficient to leave the expression $u_{4}(t)$ in the impliclt fcrm given in Eq. (24) and to solve for $u_{4}(t)$ by numerically finding the roots of the quartic equation. With this viewpolnt, it is also sufficient to represent $d u_{4} / d t$ as $\left(\mathrm{dt} / \mathrm{du}_{4}\right)^{-1}$ and then to evaluate this expression by using the numerical value of $u$ determined from the solution of the quartic. With these two considerations completed, we now have to examine the contour $c$ and examine the steps necessary to convert it to the form of Eq. (A-24). Figure A-2 Ilitstrates some of the properties of the mapping from
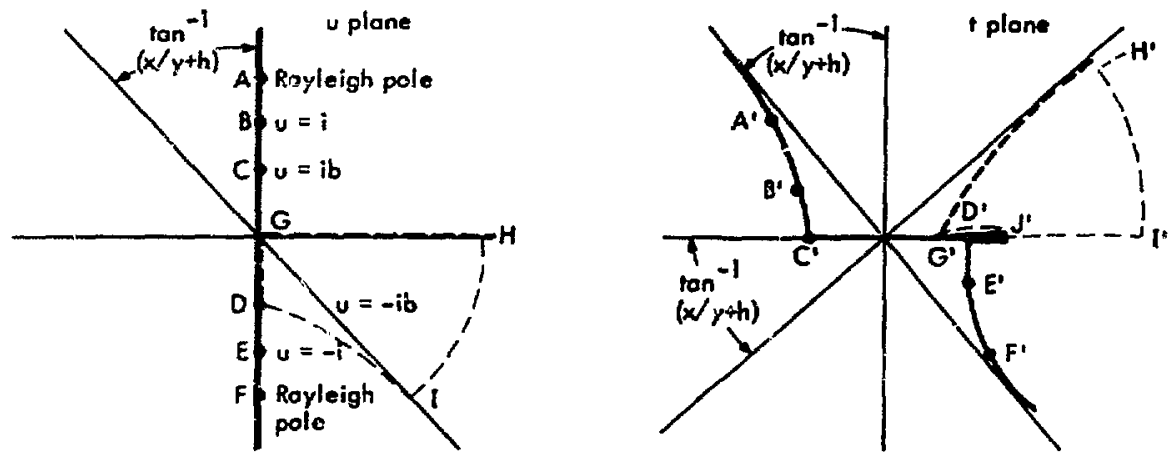

Figure A-2. The mapping from $u$ to $t$ defined by Eq. $(A-25)$.

ut to $t$ defind by Eq. $(A-25) .^{9}$ In this figure the psints $A$ and F represent the Rayleigh poles; B, C, D, and E represent branch points. The heavy dashed line in the $u$ plane is the path of integration of the integral in Eq. (A-21).

The corresponding path in the t plane does not have the form necessary to use the approach desci-ibed by Eq. $(A-24)$. We therefore seek an appropriate deformation of the contour in the u plane such that the equivalent path it the $t$ plane lies along the real axis. The integral around the closed contour GHIDG encloses no singularities, and hence equals zero as a consequence of caucry's theorem. In the limit as $\mathrm{H}$ and $I$ move away from the origin in such a way that $\left|u_{4}\right|$ approaches infinity, the integrand goes to zero in such a way that the integral from I to $H$ contributes zero. Thus the integral in the $u$ 
plane nescribed in Eq. (A-26) is equivalent to the integral along the pati GDI. We can readily show that at the point $G^{\prime}$. $t$ has the value $y / V_{c}+h / V_{s^{*}}$ Thus we have the result

$$
s_{x 4}=-\frac{2 a}{V_{s}} \operatorname{Rp} \int_{\frac{y}{V_{s}}+\frac{h}{v_{s}}}^{\infty} u_{4}^{2} \frac{\left(1+2 u_{4}^{2}\right)}{\Delta_{s}\left(b, u_{4}^{2}\right)} e^{-p t}\left[\frac{d t}{d u_{4}}\right]^{-1} d t .
$$

This is similar to the form of Eq. (25). Therefore, we he:ve

$$
\begin{array}{rlrl}
s_{x 4} & =-\frac{2 a}{v_{s}} \operatorname{Re}\left\{u_{4}^{2} \frac{\left(1+2 u_{4}^{2}\right)}{\Delta_{s}\left(b, u_{4}^{2}\right)}\left(\frac{d t}{d u_{4}}\right)^{-1}\right\} \quad t>\frac{h}{V_{s}}+\frac{y}{v_{c}} \\
& =0 & t<\frac{h}{v_{s}}+\frac{y}{v_{c}}
\end{array}
$$

We can further modify this result in the following way: The poirt $\mathrm{J}$ ' in the plane corresponds to the maximum real value of $t$ [(Eq. 25)] reached by values of $u_{4}$ on the imaginary axis. This can easily be seen by letting $u_{4}=-i v$ and then considering the derivative of $t$ with respect to $v$ set equal to zero. The value of $t\left(t=t_{J}\right)$ defined in this way corresponds to the time for a wave to travel from the source to the surface as a shear wave and then from the surface to the field point as a compressional wave (see Cagniard, 5 . 56, for a simllar discussion related to the compressional sourcel. In the range $h / v_{b}+y / v_{c}<t<t_{j}, u_{4} 1 s$ imaginary as is $d u_{4} / d t$. Thus the real part of expression $(A-28)$ is zero in this range. Therefore, we have

$$
\begin{array}{rlrl}
s_{X 4} & =-\frac{2 a}{V_{S}} \operatorname{Re}\left[u_{4}^{2} \frac{\left(1+2 u_{4}^{2}\right)}{\Delta_{S}\left(b, u^{2}\right)}\right] & t>t_{J} \\
& =0 & & t<t_{J}
\end{array}
$$

We t:an follow a similar procedure for the third term in $s_{x}[E q .\{21\}]$ and obtain the result corresponding to $\mathrm{Eq} .(\mathrm{A}-2 \mathrm{~B})$ as

$$
\begin{array}{rlrl}
s_{x 3} & =\frac{4 a b}{V_{s}} \operatorname{Re}\left[u_{3}^{2} \frac{\sqrt{1+u_{3}^{2} / b^{2}} \sqrt{1+u_{3}^{2}}}{\Delta_{s}\left(b, u_{3}^{2}\right)} \frac{d u_{3}}{J t}\right] & t>\frac{h+y}{V_{s}} \\
& =0 & t<\frac{h+y}{V_{s}}
\end{array}
$$


where

$$
\begin{aligned}
& u_{3}=u_{3}(t)=\frac{v_{s}}{r_{+}^{2}}\left[-i x t+(h+y)\left(t^{2}-\frac{r_{+}^{2}}{v_{s}^{2}}\right)^{1 / 2}\right] \\
& \frac{d u_{3}}{d t}=\frac{v_{s}}{r_{+}^{2}}\left[-i x+\frac{(h+y) t}{\left(t^{2}-r_{+}^{2} / v_{s}^{2}\right)^{1 / 2}}\right] \\
& r_{+}^{2}=k^{2}+(y+h)^{2} .
\end{aligned}
$$

In this case, the value for $s_{x 3}$ corresponding to $t_{J}$, for $s_{x 4}$ is $t=r_{+} / V_{s^{*}}$. The range $(h+y) / V_{s} \leq t \leq r_{+} / V_{s}$ corresponds to $u_{3}$ imaginary in the interval 0 to $-i x / r_{+*}$ We have to consider two cases in order to make a transition similar to the one we made in going from Eq. $(A-28)$ to $(A-29)$. Mathematically, this situation arises because the value of $u$ corresponding to $t=r_{\downarrow} / V_{s}$ may be either above or below -ib on the imaginary axis, and this will determine whicther $\sqrt{1+u_{3}^{2} / b^{2}}$ is real or imaginary. Physically, we will see that this corresponds to whether the angle of incidence is less or greater than the critical angle. Although the same radical appears in a term $s_{y 4^{\prime}}$ the value of $u_{4}$ corresponding to $t_{J}$, is always above -ib on the imaginary axis since by definition $t_{J}$, corresponds to the maximum positive real value of $t$ for values of $u_{4}$ on the imaginary axis and $t$ is not real for $u$ on the imaginary axis at values below - ib.

We now consider the two cases for $\mathrm{s}_{\mathrm{x} 3}$ [the third term in Eq. $\{\mathrm{A}-21$ )].

1. $x / r_{+} \leq b=v_{s} / v_{p},\left(h+y / v_{s} \leq t \leq r_{+} / v_{s}\right.$, i. e., the angle of incidence is less

In this case, the radicals $\sqrt{1+u_{3}^{2} / b^{2}}$ and $\sqrt{1+u_{3}^{2}}$ are real and $u_{3}$ and $d u_{3} / d t$ are imaginary. Thus the value of $s_{x 3}$ is zero in this range.

2. $x / r_{+}>b=v_{s} / v_{p^{\prime}}\left(h+y / v_{s} \leq t \leq r_{+} / v_{s:} i_{\text {than the critical angle. }}^{\text {the angle of incidence is greater }}\right.$

In this case, for $(h+y) / v_{s} \leq x / v_{c}+\sqrt{1-b^{2}}(h+y) / v_{s} \leq t \leq r_{+} / v_{s}, \sqrt{1+u_{3}^{2}}$ is real, and $\sqrt{1+u_{3}^{2} / b^{2}}, u_{3}$, and $d u_{3} / d t$ are imaginary. Thus $s_{x 3}$ is non-zero in this range.

Physically, we can interpret these results as follows: An $S$ wave striking a free surface generates a critically reflected compressional wave for angles of incidence greater than $\sin ^{-1} b$. If the field point is such that the reflected shear wave which 
arrives there struck the surface at less than the critical angle, then the contribution from the third term in Eqs. (21) and (22) begins at the time of arrival of the reflerted shear wave. If the field point is such that the angle for the reflected shear wave is greater than the critical angle, the contribution from the third term begins at a time before the arrival of the shear wave and represents a conical wave as well as a correction to the shear wave. There is no corresponding phenomenon for the compressional wave suurce.

In summary, the first two terms in Eqs. (21) and (22) represent he direct shear wave from the source and the shear wave from an image source located at $(0,-h)$. The third term represents a correction to the image term to account for the energy converted into compressional energy. This term includes the conical wave when appropriate. The divergence of the displacement arising from the third term is zero in all cases, and hence this term represents distortional energy in which there is no change in volume. The fourth term describes the compressional wave generated by the shear wave striking the free surface. 


\section{Appendix B \\ Energy Considerations Related to the Source Term}

1. The Radiated Energy

The instantaneous power $(\dot{W})$ transmitted across a surface $S$ is given by

$$
\dot{W}=-\int_{S} t_{J}^{i} \dot{S}^{j} d_{i}
$$

where

$$
\begin{aligned}
& \mathrm{t}_{j}^{\mathrm{i}}=\text { the mixed components of the second rank stress tensor, } \\
& \dot{s}^{j}=\text { the contravariant component of the particle velocity on the surface, } \\
& \mathrm{dS}_{\mathrm{i}}=\nu_{i} \mathrm{dS} \\
& \nu_{i}=\text { the covariant component of the normal to the surface, } \\
& \mathrm{dS}_{\mathrm{i}}=\text { the element of surface area. }
\end{aligned}
$$

Using the unnormalized forms of the first terms in Eqs. (1) and (2), we see that we can write the displacement due to the compressional source in an infinite medium as

$$
\vec{s}_{c}=\frac{a t}{2 r \sqrt{t^{2}-\frac{r^{2}}{v_{c}^{2}}}} \vec{u}_{r^{2}}=s_{c} \vec{u}_{r}
$$

and the corresponding displacement due to the shear source as

$$
\vec{s}_{\vec{s}}=\frac{\text { at }}{2 r \sqrt{t^{2}-\frac{r^{2}}{V_{s}^{2}}}} \vec{u}_{\phi}=s \vec{s}_{\phi}
$$

where

$$
\begin{aligned}
\mathbf{r}= & \text { the distance from the axis of the cylindrical source to the field } \\
& \text { point, } \\
\mathbf{t} & \text { time, } \\
\vec{u}_{\mathbf{u}}= & \text { unit vector in the outward radial direction from the axis, } \\
\vec{u}_{\phi}= & \text { unit vector corracponding to the vector in the direction of increas- } \\
& \text { ing } \phi \text { in a cylindrical coordinate system }(r, z, \phi) \text { where the } z \text { axis } \\
& \text { coincides with the line source. } \\
v_{c}, v_{s}= & \text { the compressional and shear wave velocities respectively. }
\end{aligned}
$$

We want to consider the energy flowing through a cylinder of radius $r$ about the line source. In this case, $v_{1}=1, v_{2}=v_{3}=0$. That is, the normal to the cylinder is in the radial direction. For the compressional source, 


$$
\dot{s}^{1} \neq \frac{\partial s c}{\partial t}=\frac{\partial}{\partial t}\left(\frac{a t}{2 r \sqrt{t^{2}-r^{2} / v_{c}^{2}}}\right), \dot{s}^{2}=\dot{s}^{3}=0 .
$$

Thus for the compressional source, the flow rate becomes

$$
-\int_{S}^{1} \frac{\partial s_{c}^{1}}{\partial t} d S
$$

Since we have axial symmetry and the surface is a concentric cylinder about the axis, this integral reduces to

$$
\dot{w}_{c}=-2 \pi r t_{1}^{1} \frac{\partial s}{\partial t}
$$

Similarly, for the shear source we have

$$
\dot{w}_{s}=-2 r^{\prime} t_{2}^{1} \frac{1}{=}\left(\frac{\partial s_{s}}{\partial t}\right) \text {. }
$$

where we have used tlat fact that $\dot{s}^{2}=\frac{1^{\partial s} s}{r \partial t}, \dot{s}^{1}=\dot{s}^{3}=0$.

If we replace stresses in terms of strains, we have

$$
\dot{W}_{c}=-2 \pi r \rho\left[v_{c}^{2}\left(\frac{\partial s_{c}}{\partial r}+\frac{s_{c}}{r}\right)-2 v_{s}^{2} \frac{s_{c}}{r}\right] \frac{\partial s_{c}}{\partial t}
$$

and

$$
\dot{W}_{s}=-2 \pi r^{2} \rho V_{s}^{2}\left[\frac{\partial}{\partial r}\left(\frac{s}{r}\right)\right] \frac{\partial s}{\partial t} .
$$

We can then substitute the forms for $s_{s}$ and $s_{c}$ from Eqs. (B-2) and (B-3) and obtain $\dot{w}_{c}$ and $\dot{w}_{s}$ in terms of $r$ and $t$.

In order to find the energy nux through the surface, we have to iniegrate the two rate terms $(B-7)$ and $(B-8)$. Since the two sources have different decay rates, we choose the intervals of integration such that the displacements of the two sources will have decayed by the same amounts. For a given value of $r$, we then choose

$$
\frac{r+t}{v_{c}}<t<\frac{r+1}{V_{c}}
$$

for the compressional scurce, and 


$$
\frac{r+c}{v_{s}}+t-\frac{r+1}{v_{s}}
$$

for the shear source. Having performed the integrations and considering the far field radiation such that $r \rightarrow \infty$, we obtain:

$$
\underset{\substack{L \\ \frac{r+c}{V_{c}}}}{\int_{c}^{\frac{r+1}{V_{c}}}} \dot{W}_{s} d t=+\frac{\pi \rho v_{p}^{2}}{8}\left(\frac{1}{c^{2}}-\frac{1}{1^{2}}\right)
$$

and

$$
\underset{\substack{L \rightarrow \infty \\ \frac{r+\epsilon}{V_{s}}}}{\int_{\frac{r+1}{V_{s}}}} \dot{w}_{s} d t=+\frac{\pi \rho V_{s}^{2}}{8}\left(\frac{1}{\epsilon^{2}}-\frac{1}{l^{2}}\right) .
$$

These results give the amount of propagating energy radiated into the tar field by the two sources in the scaled time intervals. We see that the compressional source radiates $v_{p}^{2} / v_{s}^{2}$ the amount of energy that the shear source radiates.

2. The Residual Strain Energy

In a somewhat similar manner we can consider the residual strain energy per unit volume (E). This is given by

$$
E=\stackrel{L}{t \rightarrow \infty}^{\frac{1}{2}} t^{i j} \mathbf{c}_{i j} .
$$

where

$$
\begin{aligned}
& t^{i j}=\text { the contravariani components of the stress tensor. } \\
& c_{i j}=\text { the covariant components of the strain tensor. }
\end{aligned}
$$

with

$$
t^{i j}=\lambda g^{i j} 0+2 G g^{i r} g^{j s} \text { rs }
$$

where

$$
\begin{aligned}
& \mathbf{g}^{\mathbf{i j}}=\text { the associated metric tensor, } \\
& \lambda, G=\text { Lame's constants, } \\
& 0 \quad=\mathbf{c}^{i} \text {. }
\end{aligned}
$$

In the limit as $t \rightarrow \infty$.

$$
L_{t \rightarrow \infty} \vec{s}_{c}=\frac{a}{2 t} \vec{u}_{r}
$$


and

$$
L_{i \rightarrow \infty} \vec{s}_{s}=\frac{a}{2 r} \vec{u}_{b}
$$

Substituting these forms into standard forms for the strain tensor, we ubtain

$$
F_{c}=\frac{G^{2}}{2} \frac{1}{s^{4}}=E_{s} \text {. }
$$

If we compute the total residual strain energy per unit length in the region $r=c$ to $r=b$ where $b \rightarrow \infty$, we find that the total residual strain energy per unit length is given by

$$
\frac{x^{2}-\mu}{2} \frac{1}{c^{2}}
$$

for both sources. Thus the static characteristics of two sources are the same. 


\section{Appendix C \\ The Energy Radiated in the Form of Rayleigh Waves}

Since Rayleigh waves propagate parallel to the iree surface, we may consider the energy radiated in this type of wave for the line sources by considering the energy flux through a plare perpendicular to the $x$ axis (see Fig. 1). We can follow the approach used in Part 1 of Appendix B.

The displacements due to the Rayleigh wave in the far field have been obtained by L_proood. ${ }^{15}$ Lapwood's source functions differ from the present ones onig by a constant factor of 4 related to the form chosen for the source representation. The forms given by Lapwood for the displacement due to the Rayleigh wave are (when divided by 4 to agree with our source):

1. For the compressional source,

$$
\begin{aligned}
& S_{x}=\frac{A_{P}}{4 \pi \gamma} \frac{T}{p^{2}+T^{2}}-\frac{A_{R}^{\prime}}{4 \pi \gamma_{\beta}}-\frac{T}{p^{\prime 2}+T^{2}} \\
& S_{y}=\frac{-A_{R}}{4 \pi} \frac{p}{\gamma_{\alpha}} \frac{p}{p^{2}+T^{2}}+\frac{A_{R}}{4 \pi \gamma} \frac{p^{\prime}}{p^{\prime 2}+T^{2}} .
\end{aligned}
$$

2. For the shear source,

$$
\begin{aligned}
& S_{x}=\frac{A^{\prime} R}{4 \pi \gamma} \frac{q^{\prime}}{q^{\prime 2}+T^{2}}-\frac{A_{R}}{4 \pi \gamma_{\alpha}} \frac{q}{q^{2}+T^{2}} \\
& S_{y}=\frac{A^{\prime} R}{4 \pi \gamma_{\alpha}} \frac{T}{q^{\prime 2}+T^{2}}-\frac{A_{R} \gamma_{B}}{4 \pi \gamma \gamma_{\alpha}} \frac{T}{q^{2}+T^{2}} .
\end{aligned}
$$

where

$$
\begin{aligned}
& \gamma=\text { Mayleigh wave velocity } \\
& \gamma_{a}=\frac{\gamma v_{c}}{\sqrt{v_{c}^{2}-\gamma^{2}}} \text {. } \\
& \mathrm{T}=\mathrm{t}-\frac{\mathrm{x}}{\gamma} \\
& \mathbf{p}=\frac{\mathbf{h}+\mathbf{z}}{\gamma_{\alpha}} \cdot \quad \mathbf{p}^{\prime}=\frac{\mathbf{h}}{\gamma_{\alpha}}+\frac{\mathbf{z}}{\gamma_{\beta}} . \\
& q=\frac{h+z}{\gamma^{2}}, \quad q^{\prime}=\frac{h}{\gamma_{\beta}}+\frac{z}{\gamma_{\alpha}} \text {. } \\
& A_{R}=t \pi \gamma_{\alpha}\left(\gamma_{\alpha}^{2}+\gamma_{\beta}^{2}+2 \gamma-4 \gamma_{\alpha} \gamma_{\beta}+\frac{2^{2}}{v_{s}^{2}} \gamma_{\alpha} \gamma_{\beta}\right)^{-1} . \\
& -43-
\end{aligned}
$$




$$
A_{R}^{\prime}=\frac{1}{2} \gamma \gamma_{\beta}\left(\frac{2}{\gamma^{2}}-\frac{1}{v_{s}^{2}}\right) A_{R}
$$

Carrying out operations similar to those of section $B$, we find fafter considerable algebra) that the total rudiated energy per unit length (i. e., in both dircctions from the source) from the compressional source is

$\frac{\rho A_{R}^{2}}{16^{2} \pi h^{2} \gamma^{3} \gamma_{\beta}^{2}}\left(-8 \gamma^{4} \gamma_{\alpha} \gamma_{\beta}-4 \gamma^{2} \gamma_{\alpha}^{3} \gamma_{\beta}+6 \gamma^{2} \gamma_{\alpha}^{2} \gamma_{\beta}^{2}+7 \gamma^{4} \gamma_{\alpha}^{2}-\gamma_{\alpha}^{2} \gamma_{\beta}^{4}\right)$

and the corresponding energy for the shear source is

$$
\begin{array}{r}
\frac{\rho A_{R}^{2}}{16^{2}{ }_{\pi}^{2} \gamma^{3} \gamma_{\alpha}^{2}}\left(-\gamma_{\beta}^{4} \gamma_{\alpha}^{3}-2 \gamma^{2} \gamma_{\alpha}^{3} \gamma_{\beta}^{2}-\gamma^{4} \gamma_{\alpha}^{3}-9 \gamma^{2} \gamma_{\beta}^{5}-8 \gamma^{4} \gamma_{\beta}^{3}\right. \\
\left.+8 \gamma_{\beta}^{4} \gamma^{2} \gamma_{\alpha}+8 \gamma_{\beta}^{2} \gamma^{4} \gamma_{\alpha}\right)
\end{array}
$$

where $\rho$ is the density. 


\section{Appendix D \\ Energy Partition}

The uncrease in the total energy per unit volume $\left(E_{q}\right)$ due to the suurce can be considered as the sum of the kinctic encrgy per unit volume (X. E.) and the increase in the internal chergy per unit volume $\left(F_{i}\right)$

$$
\text { i. o., E. }=K . E+E_{1} .
$$

If we neglect bemperature offects, we can replace the increase in the internal energy by the increase in the strain energy per unit volusie (W). After Fung, ${ }^{13}$ (Ch. 12). we may write

$$
W=\frac{1}{2} \mathrm{Ke}^{2}+2 \mathrm{GJ} \mathrm{J}_{2}
$$

We also have the kinetic energy per unit volume 28

$$
\text { K. } \mathrm{E}:=\frac{1}{2} \dot{\vec{s}}^{2} \text {. }
$$

where

$$
\begin{aligned}
& k \text { = bulk modulus, } \\
& G \text { = shesir modulus, } \\
& e_{i j}=\frac{i}{2}\left(\frac{\partial s_{i}}{\partial x^{j}}+\frac{\partial s_{j}}{\partial x^{i}}\right) \\
& s_{1}=s_{x} \\
& s_{2}=s_{y} \\
& 8=e_{i i}=e_{11}+e_{22}+433 \\
& J_{2}=\frac{I}{2}\left(e_{i j}-\frac{I}{2} b_{i j} c\right)\left(e_{i j}-\frac{1}{3} \delta_{i j} c\right) \\
& \delta_{i j}=\text { Kronecker delta, } \\
& \dot{s}=\frac{\partial \vec{s}}{\partial t} \text {. }
\end{aligned}
$$

If we consider the strain in an element of volume to be coniposted of a change in "rolume due to a hydrostatic stress together with a distortion of shape due to devialoric stresses, then we can identily the first term in $W$ with the change in volume and the second term with the distortion of the shape of the eiement. Thus we have the total energy per unit volume as:

$$
E_{T}=\frac{1}{2} \rho \dot{\vec{s}}^{2}+\frac{1}{2} K e^{2}+G\left(e_{i j}-s_{i j} \frac{e}{3}\right)\left(e_{i j}-\delta_{i j} \frac{e}{3}\right) .
$$


The dimensionless forms given in Eqs. (1) and (2) are functions of the variables

$$
E_{3}=\frac{x}{h}, \zeta=\frac{y}{h}, \eta \text { or } r_{i}^{\prime}=\frac{V t}{h}
$$

where $v=v_{c}$ or $v_{s}$ for $n$ and $n$ ' respectively. We have

$$
\begin{aligned}
& \frac{\partial}{\partial t}=\frac{d r}{d t} \frac{a}{\partial \eta}=\frac{V}{h} \frac{a}{\partial \eta} . \\
& \frac{\partial}{\partial x} \approx \frac{d \xi}{d x} \frac{\partial}{\partial \xi}=\frac{1}{h} \frac{\partial}{\partial \xi} . \\
& \frac{\partial}{\partial y}=\frac{d}{d y} \frac{\partial}{\partial \zeta}=\frac{1}{h} \frac{\partial}{\partial \zeta} .
\end{aligned}
$$

Thus we can express Eq. (D-4) in terms of Eqg. (1) and (2) and the variables $E$, , $n$

$$
\begin{aligned}
& \frac{\partial \vec{y}}{\partial t}=\frac{V}{h^{2}}\left[\frac{\partial\left(h s_{x}\right)}{\partial \eta} \vec{e}_{1}+\frac{\partial\left(h s_{y}\right)}{\partial \eta} \vec{e}_{c}\right] \\
& \frac{\partial s_{1}}{\partial x^{\prime}}=\frac{1}{h^{2}} \frac{\partial\left(h s_{x}\right)}{\partial t}, \quad \frac{\partial s_{1}}{\partial x^{2}}=\frac{1}{h^{2}} \frac{\partial\left(h s_{x}\right)^{2}}{\partial \zeta} . \\
& \frac{\partial s_{2}}{\partial x^{2}}=\frac{1}{h^{2}} \frac{\partial\left(h s_{y}\right)}{\partial \xi}, \quad \frac{\partial s_{2}}{\partial x^{2}}=\frac{1}{h^{2}} \frac{\partial\left(h s_{y} y^{\prime}\right.}{\partial \zeta} .
\end{aligned}
$$

If we substitute these forms into $\mathrm{Eq}$. (D-4) we can obtain

$$
\begin{aligned}
& \frac{h_{1}^{4} E_{r} r}{\sigma}=\frac{1}{2} c\left\{\left[\frac{\partial\left(h s_{x} \eta\right.}{\partial \eta}\right]^{2}+\left[\frac{\partial\left(h s_{y}{ }^{2}\right.}{\partial \eta}\right]^{2}+\frac{1+\sigma}{3(1+2 \sigma)} e^{-2}\right.
\end{aligned}
$$

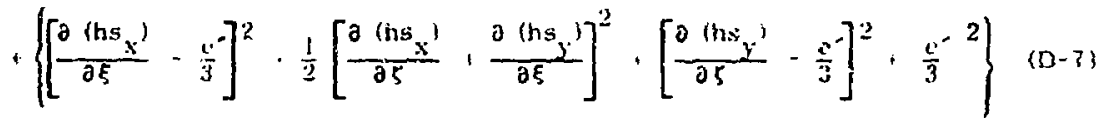

where we have used

$$
\begin{aligned}
\sigma & =\text { Poisson's ratio } \\
c & =\frac{2-2 \sigma}{1-2 \sigma} \text { if } v=v_{p} . \\
& =1 \quad \text { if } v=v_{s} .
\end{aligned}
$$




$$
\begin{aligned}
& e^{-}=\frac{\partial\left(h s_{x}\right)}{\partial \xi}+\frac{\partial\left(h s_{y}\right)}{\partial \zeta} . \\
& K=\frac{2(1+\sigma)}{3(1-2 \sigma)} .
\end{aligned}
$$

It is of interest to note that a compressional wave, i. e., one whose displacement $\vec{s}$ is such that $\nabla \times \vec{B}=0$, containg both types of internal energy, whereas the shear wave, 1. e., one whose displacement is such that $\nabla \cdot s=0$, contains only distortional energy. 


\section{References}

1. H. C. Rodean, Lawrence Livermore Laboratory, personal communication.

2. H. Nakano, "On Rayleigh Waves," Japan 3. Astron,Geophys. , 2, 233 (1925).

3. W. M. Ewing, W. S. Jardetzky, and F. Press, Elastic Waves in Layered Media, (McGraw-Hill, Niew York, 1957).

4. W. W. Garvin, "Exact Transient Solution or the Huried Line Source Problem," Proc. Roy Soc. (London), 234A, 528 (1956).

5. L. Cagniard, Reftection and Rstraction of Progressive Selimic Waves, (trans. by

E. A. Flynn and C. H. Dix) (McGraw-Hill, New York, 1962).

6. C. H. Dix, "The Mechanism of Generation of Long Waves from Explosions," Geophysics, 20, 87 (1955).

7. H. Takeuchi and N. Kobayashi, "Wave Generations from Line Sources within the Ground," J. Phys, Earth, 3, 7 (1955).

8. N. Kobayashi and $H$, Takeuchi, "Wave Generations from l.ine Sources within the Ground." J. Phys, Eiarth, 5, 25 (1957).

9. S. Nagumo, On the Propagation of Transient Elastic Waves, Geological Survey of Japan, Report 184 (1960).

10. F. Gllbert and L. Knopoff, "The Directivity Problem for a Buried L:ne Source," Geophysicg, 26, 626 (1961).

11. E. J. Mateker, Genuration of Rayleigh Waves by Contained Explosions, Ph.D. thesis, St, I.ouis University (1964).

12. I. Tolstoy and E. U', "n, "Dispersive Properties of Stratified Elastic and Liquid Media: A Ray Ther...' Geophysics, 18, 844 (1953).

13. Y. C. Fung, The Foundations of Solid Mechanics (Prentice-Hall, Englewood Cliff, N. J. 1965).

14. A. T. deHoop, "A Modification of Cagniard's Method for Solving Seismic Pulse $\Gamma$ blems," Appl. Sci. Res., B, B, 349 (1960).

15. . 7. Lapwood, "The Disturbance Due to a Line Source in a Semi-infinite Elastic .hedium," Proc. Boy. Soc. (London), 242A 63 (1949). 
